\title{
SPICE_MARS: A Process Synthesis Framework for Membrane-Assisted Reactive Separations
}

Mohammed Sadaf Monjur, Salih Emre Demirel, Jianping Li, and M. M. Faruque Hasan* Artie McFerrin Department of Chemical Engineering, Texas A\&M University College Station, TX 77843-3122, USA.

\section{Supporting Information}

In the Supporting Information, we provide additional details on those topics that are important but could not be included in the main manuscript. Specifically, detailed description of the optimization model, reaction kinetics, reactor specifications, physical parameters of thermodynamic property models, detailed formulation of capital cost functions, and definition of selectivities and syngas ratio.

\section{Contents}

$\begin{array}{ll}\text { S1 Optimization Model } & \text { S2 }\end{array}$

$\begin{array}{ll}\text { S2 Reaction Kinetics } & \text { S16 }\end{array}$

$\begin{array}{ll}\text { S3 Reactor Specifications } & \text { S19 }\end{array}$

S4 Surrogate Models of Thermodynamic Properties $\quad$ S19

S5 in silico Validation of Thermodynamic Property Models $\quad$ S21

S6 Capital Cost Functions for Total Annual Cost (TAC) S22

$\begin{array}{ll}\text { S7 Selectivity and Syngas Ratio } & \text { S23 }\end{array}$

\section{List of Figures}

S1 Accuracy of surrogate models for enthalpy estimation for (a) $\mathrm{CO}_{2}$, (b) $\mathrm{O}_{2}$, (c) $\mathrm{N}_{2}$, (d) $\mathrm{H}_{2} \mathrm{O}$, (e) $\mathrm{CH}_{4}$, (f) $\mathrm{CO}$, (g) $\mathrm{H}_{2}$, and (h) $\mathrm{CH}_{3} \mathrm{OH}$ between temperature range of $300 \mathrm{~K}$ to $1600 \mathrm{~K} \ldots \ldots \ldots$. . . . . . . . . . . . . . . . . S21

S2 Methanol synthesis process simulation, (a) $1 \times 13$ building block superstructure considered in SPICE_MARS, and (b) corresponding ASPEN PLUS ${ }^{\circledR}$ flow sheet. . . . . S21 


\section{List of Tables}

S1 Reaction rate and adsorption equilibrium constants for methanol synthesis reactions. S17

S2 Reaction equilibrium constants for methanol synthesis reactions. . . . . . . . . . S S17

S3 Arrhenius parameters and effectiveness factors for POM reactions. . . . . . . . . S18

S4 Van't Hoff parameters for the POM adsorption reactions. . . . . . . . . . . . . S18

S5 Reaction equilibrium constants for POM reactions. . . . . . . . . . . . . . . S18

S6 Design parameters of methanol synthesis reactor. . . . . . . . . . . . . . . S19

S7 Design parameters of POM reactor. . . . . . . . . . . . . . . . S19

S8 Vapor phase specific enthalpy parameters. . . . . . . . . . . . . . S20

S9 Comparison between SPICE_MARS and ASPEN PLUS ${ }^{\circledR}$ simulation of isothermal and adiabatic methanol synthesis reactor. . . . . . . . . . . . . . S22

\section{S1 Optimization Model}

In this section, we provide the detailed mathematical model for synthesis and intensification of the membrane-assisted reactive separation system. To distinguish the equations of the manuscript, here every equations are numbered with an additional ' $\mathrm{S}$ '. Therefore, when a equation is written without ' $S$ ', it refers to the equation of the main manuscript.

\section{Flow Directions:}

The disjunction for unidirectional flow in Eq. 8 is written with big-M constraints as follows,

$$
\begin{gathered}
F P_{i, j, k} \leq F U_{i, j, k} z_{i, j}^{\text {Fplus }}, \quad i \in \mathcal{I}, j \in \mathcal{J}, k \in \mathcal{K}, \\
F N_{i, j, k} \leq F U_{i, j, k}\left(1-z_{i, j}^{\text {Fplus }}\right), \quad i \in \mathcal{I}, j \in \mathcal{J}, k \in \mathcal{K}, \\
R P_{i, j, k} \leq R U_{i, j, k} z_{i, j}^{\text {Rplus }}, \quad i \in \mathcal{I}, j \in \mathcal{J}, k \in \mathcal{K}, \\
R N_{i, j, k} \leq R U_{i, j, k}\left(1-z_{i, j}^{\text {Rplus }}\right), \quad i \in \mathcal{I}, j \in \mathcal{J}, k \in \mathcal{K} .
\end{gathered}
$$

Here, $F U_{i, j, k}$ and $R U_{i, j, k}$ are the upper bound on the flow rates of component $k$ in horizontal and vertical direction, respectively.

The composition of unrestricted flows has to be equal to the composition of its source block (Eq. 11). To ensure that, we wrote,

$$
\begin{aligned}
& F P_{i, j, k} \leq y_{i, j, k} \sum_{k^{\prime} \in \mathcal{K}} F P_{i, j, k^{\prime}}+F U\left(1-z_{i, j}^{u n F}\right), \quad i \in \mathcal{I}, j \in \mathcal{J}, k \in \mathcal{K}, \\
& F P_{i, j, k} \geq y_{i, j, k} \sum_{k^{\prime} \in \mathcal{K}} F P_{i, j, k^{\prime}}-F U\left(1-z_{i, j}^{u n F}\right), \quad i \in \mathcal{I}, j \in \mathcal{J}, k \in \mathcal{K},
\end{aligned}
$$




$$
\begin{gathered}
F N_{i, j, k} \leq y_{i, j+1, k} \sum_{k^{\prime} \in \mathcal{K}} F N_{i, j, k^{\prime}}+F U\left(1-z_{i, j}^{u n F}\right), \quad i \in \mathcal{I}, j \in \mathcal{J}, k \in \mathcal{K}, \\
F N_{i, j, k} \geq y_{i, j+1, k} \sum_{k^{\prime} \in \mathcal{K}} F N_{i, j, k^{\prime}}-F U\left(1-z_{i, j}^{u n F}\right), \quad i \in \mathcal{I}, j \in \mathcal{J}, k \in \mathcal{K}, \\
R P_{i, j, k} \leq y_{i, j, k} \sum_{k^{\prime} \in \mathcal{K}} R P_{i, j, k^{\prime}}+R U\left(1-z_{i, j}^{u n R}\right), \quad i \in \mathcal{I}, j \in \mathcal{J}, k \in \mathcal{K}, \\
R P_{i, j, k} \geq y_{i, j, k} \sum_{k^{\prime} \in \mathcal{K}} R P_{i, j, k^{\prime}}-R U\left(1-z_{i, j}^{u n R}\right), \quad i \in \mathcal{I}, j \in \mathcal{J}, k \in \mathcal{K}, \\
R N_{i, j, k} \leq y_{i+1, j, k} \sum_{k^{\prime} \in \mathcal{K}} R P_{i, j, k^{\prime}}+R U\left(1-z_{i, j}^{u n R}\right), \quad i \in \mathcal{I}, j \in \mathcal{J}, k \in \mathcal{K}, \\
R N_{i, j, k} \geq y_{i+1, j, k} \sum_{k^{\prime} \in \mathcal{K}} R P_{i, j, k^{\prime}}-R U\left(1-z_{i, j}^{u n R}\right), \quad i \in \mathcal{I}, j \in \mathcal{J}, k \in \mathcal{K},
\end{gathered}
$$

where, $F U$ and $R U$ represent the upper bound on the total flow rate allowed through a boundary in horizontal and vertical direction.

When the boundary is completely restricted, flow through that boundary is zero (Eq. 14),

$$
\begin{aligned}
& F_{i, j, k} \leq F U\left(1-z_{i, j}^{c r F}\right), \quad i \in \mathcal{I}, j \in \mathcal{J}, k \in \mathcal{K}, \\
& R_{i, j, k} \leq R U\left(1-z_{i, j}^{c r R}\right), \quad i \in \mathcal{I}, j \in \mathcal{J}, k \in \mathcal{K} .
\end{aligned}
$$

\section{Phase Determination:}

To determine the phase of a block, we define a binary variable,

$z_{i, j}^{\text {phase }}= \begin{cases}1 & \text { if block } B_{i, j} \text { is in vapor } / \text { gas phase } \\ 0 & \text { otherwise }\end{cases}$

The phase of each block is determined from the bubble and dew pressure of the mixture by

$$
\begin{gathered}
P_{i, j} \geq P_{i, j}^{b u b}-P_{i, j}^{b u b, U p} z_{i, j}^{\text {phase }}, \quad i, j \in \mathcal{L} \mathcal{B}, \\
P_{i, j} \leq P_{i, j}^{\text {dew }}+P^{\max }\left(1-z_{i, j}^{\text {phase }}\right), \quad i, j \in \mathcal{V B} .
\end{gathered}
$$

Eq. S15, ensures the pressure of a liquid block is greater than the bubble pressure of the mixture. On the other hand, when the block is in vapor or gas phase, the pressure of the block has to be lower than the dew pressure of the mixture. This is imposed by Eq. S16. However, incorporating these phase check constraints in every block of the superstructure can be computationally demanding. For that reason, these checks are allowed in certain blocks $B_{i, j}$ denoted by $\mathcal{L B}$ and $\mathcal{V} \mathcal{B}$. Bubble pressure and dew pressure are calculated by 


$$
\begin{gathered}
P_{i, j}^{b u b}=\sum_{k \in \mathcal{K}} y_{i, j, k} P_{i, j, k}^{s a t}, \quad i, j \in \mathcal{L B}, \\
P_{i, j}^{\text {dew }}=\frac{1}{\sum_{k \in \mathcal{K}} \frac{y_{i, j, k}}{P_{i, j, k}^{s a t}}}, \quad i, j \in \mathcal{V B} .
\end{gathered}
$$

Saturation pressure of each component $k$ is estimated either from Antoine equation or from temperature depended surrogate models,

$$
\begin{gathered}
P_{i, j, k}^{s a t}=\exp \left(A_{k}^{a n t}-\frac{B_{k}^{a n t}}{T_{i, j}+C_{k}^{a n t}}\right), \quad i, j \in \mathcal{L} \mathcal{B} \cup \mathcal{V B}, k \in \mathcal{K} \mathcal{A} \\
P_{i, j, k}^{s a t}=P_{k}^{a} T_{i, j}^{2}+P_{k}^{b} T_{i, j}+P_{k}^{c}, \quad i, j \in \mathcal{L} \mathcal{B} \cup \mathcal{V B}, k \in \mathcal{K} / \mathcal{K} \mathcal{A} .
\end{gathered}
$$

Here, $\mathcal{K} \mathcal{A}$ is the set of components $k$ for which saturation pressure is calculated by Antoine equation (Eq. S19). $A_{k}^{\text {ant }}, B_{k}^{\text {ant }}$, and $C_{k}^{\text {ant }}$ are the Antoine parameters and Eq. S20 represents the temperature depended quadratic surrogate model.

If a product is withdrawn from a block, the phase of the product has to be same as the phase of the block. To impose this, we write,

$$
\begin{aligned}
& z_{i, j}^{\text {phase }} \geq z_{p}^{\text {phaseprod }}-\left(1-z_{i, j, p}^{\text {product }}\right), \quad i \in \mathcal{I}, j \in \mathcal{J}, p \in \mathcal{P S}, \\
& z_{i, j}^{\text {phase }} \leq z_{p}^{\text {phaseprod }}+\left(1-z_{i, j, p}^{\text {product }}\right), \quad i \in \mathcal{I}, j \in \mathcal{J}, p \in \mathcal{P S},
\end{aligned}
$$

where, $z^{\text {phaseprod }}$ is a $0-1$ parameter, that defines the pre-specified phase of the product stream $p$.

\section{Energy Balances:}

The block energy balances are describe by Eq. 15. Based on the flow direction and the phase of the streams, the enthalpy terms are dissected in multiple parts. For instance, the enthalpy term in the horizontal direction, $E F_{i, j}$ has four terms,

$$
E F_{i, j}=\sum_{k \in \mathcal{K}} E F P_{i, j, k}^{g}+\sum_{k \in \mathcal{K}} E F P_{i, j, k}^{l}-\sum_{k \in \mathcal{K}} E F N_{i, j, k}^{g}-\sum_{k \in \mathcal{K}} E F N_{i, j, k}^{l}, \quad i \in \mathcal{I}, j \in \mathcal{J}
$$

Similarly, stream enthalpy in the vertical direction is expressed as

$$
E R_{i, j}=\sum_{k \in \mathcal{K}} E R P_{i, j, k}^{g}+\sum_{k \in \mathcal{K}} E R P_{i, j, k}^{l}-\sum_{k \in \mathcal{K}} E R N_{i, j, k}^{g}-\sum_{k \in \mathcal{K}} E R N_{i, j, k}^{l}, \quad i \in \mathcal{I}, j \in \mathcal{J}
$$

Individual stream enthalpy terms are determined from the stream flow rate, temperature, pressure, and the phase of the source block. Based on the ideal gas assumption, we use temperature depended surrogate models for estimating component enthalpies. For instance, for a gaseous streams flowing in the horizontal positive direction, the enthalpy of component $k$ in that stream is expressed by 


$$
\begin{gathered}
E F P_{i, j, k}^{g}+E F P_{i, j, k}^{g s}= \\
F P_{i, j, k}\left(H_{k}^{v a p, a} T_{i, j}^{2}+H_{k}^{v a p, b} T_{i, j}+H_{k}^{v a p, c}\right), \quad i \in \mathcal{I}, j \in \mathcal{J}, k \in \mathcal{K}, \\
\quad E F P_{i, j, k}^{g} \leq E U \times z_{i, j}^{\text {phase }}, \quad i \in \mathcal{I}, j \in \mathcal{J}, k \in \mathcal{K}, \\
\quad E F P_{i, j, k}^{g} \geq-E U \times z_{i, j}^{\text {phase }}, \quad i \in \mathcal{I}, j \in \mathcal{J}, k \in \mathcal{K}, \\
E F P_{i, j, k}^{g s} \leq E U \times\left(1-z_{i, j}^{\text {phase }}\right), \quad i \in \mathcal{I}, j \in \mathcal{J}, k \in \mathcal{K}, \\
E F P_{i, j, k}^{g s} \geq-E U \times\left(1-z_{i, j}^{\text {phase }}\right), \quad i \in \mathcal{I}, j \in \mathcal{J}, k \in \mathcal{K} .
\end{gathered}
$$

Depending on the phase of the stream, Eq. S25 assigns the enthalpy to either $E F P_{i, j, k}^{g}$ or $E F P_{i, j, k}^{g s}$, where the latter term is a slack variable. $E F P_{i, j, k}^{g s}$ becomes active when the stream is in liquid phase (Eqs. S28-S29). Likewise, component enthalpy in liquid streams is estimated as

$$
\begin{gathered}
E F P_{i, j, k}^{l}+E F P_{i, j, k}^{l s}=F P_{i, j, k}\left(H_{k}^{l i q, a} T_{i, j}^{2}+H_{k}^{l i q, b} T_{i, j}+H_{k}^{l i q, c}\right), \quad i \in \mathcal{I}, j \in \mathcal{J}, k \in \mathcal{K}, \\
E F P_{i, j, k}^{l} \leq E U \times\left(1-z_{i, j}^{\text {phase }}\right), \quad i \in \mathcal{I}, j \in \mathcal{J}, k \in \mathcal{K}, \\
E F P_{i, j, k}^{l} \geq-E U \times\left(1-z_{i, j}^{\text {phase }}\right), \quad i \in \mathcal{I}, j \in \mathcal{J}, k \in \mathcal{K}, \\
E F P_{i, j, k}^{l s} \leq E U \times z_{i, j}^{\text {phase }}, \quad i \in \mathcal{I}, j \in \mathcal{J}, k \in \mathcal{K}, \\
E F P_{i, j, k}^{l s} \geq-E U \times z_{i, j}^{\text {phase }}, \quad i \in \mathcal{I}, j \in \mathcal{J}, k \in \mathcal{K} .
\end{gathered}
$$

Similarly, enthalpies in the horizontal negative and vertical directions are expressed as

$$
\begin{gathered}
E F N_{i, j, k}^{g}+E F N_{i, j, k}^{g s}=F N_{i, j, k}\left(H_{k}^{v a p, a} T_{i, j+1}^{2}+H_{k}^{v a p, b} T_{i, j+1}+H_{k}^{v a p, c}\right), \quad i \in \mathcal{I}, j \in \mathcal{J}, k \in \mathcal{K}, \\
E F N_{i, j, k}^{g} \leq E U \times z_{i, j+1}^{\text {phase }}, \quad i \in \mathcal{I}, j \in \mathcal{J}, k \in \mathcal{K}, \\
E F N_{i, j, k}^{g} \geq-E U \times z_{i, j+1}^{\text {phase }}, \quad i \in \mathcal{I}, j \in \mathcal{J}, k \in \mathcal{K}, \\
E F N_{i, j, k}^{g s} \leq E U \times\left(1-z_{i, j+1}^{\text {phase }}\right), \quad i \in \mathcal{I}, j \in \mathcal{J}, k \in \mathcal{K}, \\
E F N_{i, j, k}^{g s} \geq-E U \times\left(1-z_{i, j+1}^{\text {phase }}\right), \quad i \in \mathcal{I}, j \in \mathcal{J}, k \in \mathcal{K}, \\
E F N_{i, j, k}^{l}+E F N_{i, j, k}^{l s}=F N_{i, j, k}\left(H_{k}^{l i q, a} T_{i, j+1}^{2}+H_{k}^{l i q, b} T_{i, j+1}+H_{k}^{l i q, c}\right), \quad i \in \mathcal{I}, j \in \mathcal{J}, k \in \mathcal{K}, \\
E F N_{i, j, k}^{l} \leq E U \times\left(1-z_{i, j+1}^{\text {phase }}\right), \quad i \in \mathcal{I}, j \in \mathcal{J}, k \in \mathcal{K}, \\
E F N_{i, j, k}^{l} \geq-E U \times\left(1-z_{i, j+1}^{\text {phase }}\right), \quad i \in \mathcal{I}, j \in \mathcal{J}, k \in \mathcal{K},
\end{gathered}
$$




$$
\begin{aligned}
& E F N_{i, j, k}^{l s} \leq E U \times z_{i, j+1}^{\text {phase }}, \quad i \in \mathcal{I}, j \in \mathcal{J}, k \in \mathcal{K}, \\
& E F N_{i, j, k}^{l s} \geq-E U \times z_{i, j+1}^{\text {phase }}, \quad i \in \mathcal{I}, j \in \mathcal{J}, k \in \mathcal{K}, \\
& E R P_{i, j, k}^{g}+E R P_{i, j, k}^{g s}=R P_{i, j, k}\left(H_{k}^{v a p, a} T_{i, j}^{2}+H_{k}^{v a p, b} T_{i, j}+H_{k}^{v a p, c}\right), \quad i \in \mathcal{I}, j \in \mathcal{J}, k \in \mathcal{K}, \\
& E R P_{i, j, k}^{g} \leq E U \times z_{i, j}^{\text {phase }}, \quad i \in \mathcal{I}, j \in \mathcal{J}, k \in \mathcal{K}, \\
& E R P_{i, j, k}^{g} \geq-E U \times z_{i, j}^{\text {phase }}, \quad i \in \mathcal{I}, j \in \mathcal{J}, k \in \mathcal{K}, \\
& E R P_{i, j, k}^{g s} \leq E U \times\left(1-z_{i, j}^{\text {phase }}\right), \quad i \in \mathcal{I}, j \in \mathcal{J}, k \in \mathcal{K}, \\
& E R P_{i, j, k}^{g s} \geq-E U \times\left(1-z_{i, j}^{\text {phase }}\right), \quad i \in \mathcal{I}, j \in \mathcal{J}, k \in \mathcal{K}, \\
& E R P_{i, j, k}^{l}+E R P_{i, j, k}^{l s}=R P_{i, j, k}\left(H_{k}^{l i q, a} T_{i, j}^{2}+H_{k}^{l i q, b} T_{i, j}+H_{k}^{l i q, c}\right), \quad i \in \mathcal{I}, j \in \mathcal{J}, k \in \mathcal{K}, \\
& E R P_{i, j, k}^{l} \leq E U \times\left(1-z_{i, j}^{\text {phase }}\right), \quad i \in I, j \in J, k \in K \\
& E R P_{i, j, k}^{l} \geq-E U \times\left(1-z_{i, j}^{\text {phase }}\right), \quad i \in \mathcal{I}, j \in \mathcal{J}, k \in \mathcal{K}, \\
& E R P_{i, j, k}^{l s} \leq E U \times z_{i, j}^{\text {phase }}, \quad i \in \mathcal{I}, j \in \mathcal{J}, k \in \mathcal{K}, \\
& E R P_{i, j, k}^{l s} \geq-E U \times z_{i, j}^{\text {phase }}, \quad i \in \mathcal{I}, j \in \mathcal{J}, k \in \mathcal{K}, \\
& E R N_{i, j, k}^{g}+E R N_{i, j, k}^{g s}=R N_{i, j, k}\left(H_{k}^{v a p, a} T_{i+1, j}^{2}+H_{k}^{v a p, b} T_{i+1, j}+H_{k}^{v a p, c}\right), i \in \mathcal{I}, j \in \mathcal{J}, k \in \mathcal{K}, \\
& E R N_{i, j, k}^{g} \leq E U \times z_{i+1, j}^{\text {phase }}, \quad i \in \mathcal{I}, j \in \mathcal{J}, k \in \mathcal{K}, \\
& E R N_{i, j, k}^{g} \geq-E U \times z_{i+1, j}^{\text {phase }}, \quad i \in \mathcal{I}, j \in \mathcal{J}, k \in \mathcal{K}, \\
& E R N_{i, j, k}^{g s} \leq E U \times\left(1-z_{i+1, j}^{\text {phase }}\right), \quad i \in \mathcal{I}, j \in \mathcal{J}, k \in \mathcal{K}, \\
& E R N_{i, j, k}^{g s} \geq-E U \times\left(1-z_{i+1, j}^{\text {phase }}\right), \quad i \in \mathcal{I}, j \in \mathcal{J}, k \in \mathcal{K}, \\
& E R N_{i, j, k}^{l}+E R N_{i, j, k}^{l s}=R N_{i, j, k}\left(H_{k}^{l i q, a} T_{i+1, j}^{2}+H_{k}^{l i q, b} T_{i+1, j}+H_{k}^{l i q, c}\right), \quad i \in \mathcal{I}, j \in \mathcal{J}, k \in \mathcal{K} \\
& E R N_{i, j, k}^{l} \leq E U \times\left(1-z_{i+1, j}^{\text {phase }}\right), \quad i \in \mathcal{I}, j \in \mathcal{J}, k \in \mathcal{K} \\
& E R N_{i, j, k}^{l} \geq-E U \times\left(1-z_{i+1, j}^{\text {phase }}\right), \quad i \in \mathcal{I}, j \in \mathcal{J}, k \in \mathcal{K}, \\
& E R N_{i, j, k}^{l s} \leq E U \times z_{i+1, j}^{\text {phase }}, \quad i \in \mathcal{I}, j \in \mathcal{J}, k \in \mathcal{K},
\end{aligned}
$$




$$
E R N_{i, j, k}^{l s} \geq-E U \times z_{i+1, j}^{\text {phase }}, \quad i \in \mathcal{I}, j \in \mathcal{J}, k \in \mathcal{K} .
$$

Enthalpy of the jump streams, external feed streams, and product outlet streams are calculated by

$$
\begin{aligned}
& E J_{i, j, i^{\prime}, j^{\prime}, k}^{g}+E J_{i, j, i^{\prime}, j^{\prime}, k}^{g s}=J_{i, j, i^{\prime}, j^{\prime}, k}\left(H_{k}^{v a p, a} T_{i, j}^{2}+H_{k}^{v a p, b} T_{i, j}+H_{k}^{v a p, c}\right), \quad i, j, i^{\prime}, j^{\prime} \in \mathcal{L} \mathcal{I} \mathcal{N} \mathcal{K}, k \in \mathcal{K}, \\
& E J_{i, j, i^{\prime}, j^{\prime}, k}^{g} \leq E U \times z_{i, j}^{\text {phase }}, \quad i, j, i^{\prime}, j^{\prime} \in \mathcal{L} \mathcal{I} \mathcal{N} \mathcal{K}, k \in \mathcal{K}, \\
& E J_{i, j, i^{\prime}, j^{\prime}, k}^{g} \geq-E U \times z_{i, j}^{\text {phase }}, \quad i, j, i^{\prime}, j^{\prime} \in \mathcal{L} \mathcal{I} \mathcal{N} \mathcal{K}, k \in \mathcal{K}, \\
& E J_{i, j, i^{\prime}, j^{\prime}, k}^{g s} \leq E U \times\left(1-z_{i, j}^{\text {phase }}\right), \quad i, j, i^{\prime}, j^{\prime} \in \mathcal{L} \mathcal{I} \mathcal{N} \mathcal{K}, k \in \mathcal{K}, \\
& E J_{i, j, i^{\prime}, j^{\prime}, k}^{g s} \leq E U \times\left(1-z_{i, j}^{\text {phase }}\right), \quad i, j, i^{\prime}, j^{\prime} \in \mathcal{L} \mathcal{I} \mathcal{N} \mathcal{K}, k \in \mathcal{K}, \\
& E J_{i, j, i^{\prime}, j^{\prime}, k}^{l}+E J_{i, j, i^{\prime}, j^{\prime}, k}^{l s}=J_{i, j, i^{\prime}, j^{\prime}, k}\left(H_{k}^{l i q, a} T_{i, j}^{2}+H_{k}^{l i q, b} T_{i, j}+H_{k}^{l i q, c}\right), \quad i, j, i^{\prime}, j^{\prime} \in \mathcal{L} \mathcal{I N} \mathcal{K}, k \in \mathcal{K}, \\
& E J_{i, j, i^{\prime}, j^{\prime}, k}^{l} \leq E U \times\left(1-z_{i, j}^{\text {phase }}\right), \quad i, j, i^{\prime}, j^{\prime} \in \mathcal{L} \mathcal{I} \mathcal{N} \mathcal{K}, k \in \mathcal{K}, \\
& E J_{i, j, i^{\prime}, j^{\prime}, k}^{l} \geq-E U \times\left(1-z_{i, j}^{\text {phase }}\right), \quad i, j, i^{\prime}, j^{\prime} \in \mathcal{L} \mathcal{I} \mathcal{N} \mathcal{K}, k \in \mathcal{K}, \\
& E J_{i, j, i^{\prime}, j^{\prime}, k}^{l s} \leq E U \times z_{i, j}^{\text {phase }}, \quad i, j, i^{\prime}, j^{\prime} \in \mathcal{L} \mathcal{I} \mathcal{N} \mathcal{K}, k \in \mathcal{K}, \\
& E J_{i, j, i^{\prime}, j^{\prime}, k}^{l s} \leq E U \times z_{i, j}^{\text {phase }}, \quad i, j, i^{\prime}, j^{\prime} \in \mathcal{L} \mathcal{I} \mathcal{N} \mathcal{K}, k \in \mathcal{K}, \\
& E M_{i, j}=\sum_{k \in \mathcal{K}} \sum_{f \in \mathcal{F} \mathcal{S}} M_{i, j, k, f}\left(H_{k}^{f e e d, a}\left(T_{f}^{f e e d}\right)^{2}+H_{k}^{f e e d, b} T_{f}^{f e e d}+H_{k}^{f e e d, c}\right), \quad i \in \mathcal{I}, j \in \mathcal{J}, \\
& E N_{i, j}^{g}+E N_{i, j}^{g s}=\sum_{k \in \mathcal{K}} \sum_{p \in \mathcal{P} \mathcal{S}} N_{i, j, k, p}\left(H_{k}^{v a p, a} T_{i, j}^{2}+H_{k}^{v a p, b} T_{i, j}+H_{k}^{v a p, c}\right), \quad i \in \mathcal{I}, j \in \mathcal{J}, \\
& E N_{i, j}^{g} \leq E U \times z_{i, j}^{\text {phase }}, \quad i \in \mathcal{I}, j \in \mathcal{J} \\
& E N_{i, j}^{g} \geq-E U \times z_{i, j}^{\text {phase }}, \quad i \in \mathcal{I}, j \in \mathcal{J} \\
& E N_{i, j}^{g s} \leq E U \times\left(1-z_{i, j}^{\text {phase }}\right), \quad i \in \mathcal{I}, j \in \mathcal{J} \\
& E N_{i, j}^{g s} \geq-E U \times\left(1-z_{i, j}^{\text {phase }}\right), \quad i \in \mathcal{I}, j \in \mathcal{J}, \\
& E N_{i, j}^{l}+E N_{i, j}^{l s}=\sum_{k \in \mathcal{K}} \sum_{p \in \mathcal{P} \mathcal{S}} N_{i, j, k, p}\left(H_{k}^{l i q, a} T_{i, j}^{2}+H_{k}^{l i q, b} T_{i, j}+H_{k}^{l i q, c}\right), \quad i \in \mathcal{I}, j \in \mathcal{J},
\end{aligned}
$$




$$
\begin{gathered}
E N_{i, j}^{l} \leq E U \times\left(1-z_{i, j}^{\text {phase }}\right), \quad i \in \mathcal{I}, j \in \mathcal{J}, \\
E N_{i, j}^{l} \geq-E U \times\left(1-z_{i, j}^{\text {phase }}\right), \quad i \in \mathcal{I}, j \in \mathcal{J}, \\
E N_{i, j}^{l s} \leq E U \times z_{i, j}^{\text {phase }}, \quad i \in \mathcal{I}, j \in \mathcal{J}, \\
E N_{i, j}^{l s} \geq-E U \times z_{i, j}^{\text {phase }}, \quad i \in \mathcal{I}, j \in \mathcal{J} .
\end{gathered}
$$

Enthalpy change due to chemical reactions are estimated by

$$
E G_{i, j}=\sum_{\left(k^{*}, r\right) \in \mathcal{K} \mathcal{R}} \sum_{c \in \mathcal{R} \mathcal{X} \mathcal{C}} R e_{i, j, k^{*}, r, c} Q_{r}^{r x n}, \quad i \in \mathcal{I}, j \in \mathcal{J}
$$

Here, $Q_{r}^{r x n}$ is the heat of reaction for reaction $r$ based on per mole consumption of key reactant and the set $\mathcal{K} \mathcal{R}$ defines the relationship between key reactant $k^{*}$ and reaction $r$.

The compression $\left(W_{i, j}^{c o m p}\right)$ and expansion $\left(W_{i, j}^{\text {exp }}\right)$ work are calculated by summing the work requirement for all the incoming gas streams. That includes horizontal and vertical streams from all four directions, the incoming fresh feeds, and the jump streams. So we write,

$$
\begin{aligned}
& W_{i, j}^{c o m p}=W_{i, j}^{c o m p, F P}+W_{i, j}^{c o m p, F N}+W_{i, j}^{c o m p, R P}+W_{i, j}^{c o m p, R N}+\sum_{f \in \mathcal{F} \mathcal{S}} W_{i, j, f}^{c o m p, F S} \\
& +\sum_{i^{\prime}, j^{\prime} \in \mathcal{L} \mathcal{I} \mathcal{N K}} W_{i, j, i^{\prime}, j^{\prime}}^{c o m p, J}, \quad i \in \mathcal{I}, j \in \mathcal{J} \\
& W_{i, j}^{e x p}=W_{i, j}^{e x p, F P}+W_{i, j}^{e x p, F N}+W_{i, j}^{e x p, R P}+W_{i, j}^{e x p, R N}+\sum_{f \in \mathcal{F} \mathcal{S}} W_{i, j, f}^{e x p, F S} \\
& +\sum_{i^{\prime}, j^{\prime} \in \mathcal{L} \mathcal{I} \mathcal{N K}} W_{i, j, i^{\prime}, j^{\prime}}^{e x p, J F}, \quad i \in \mathcal{I}, j \in \mathcal{J}
\end{aligned}
$$

To estimate the required work due to pressure change, we use pressure ratio. The pressure ratio in the horizontal direction is denoted by the variable $P R_{i, j}^{F}$ and defined by the ratio of the pressure of block $B_{i, j+1}$ to the block $B_{i, j}$. When the common block boundary between these two blocks is unrestricted i.e. $z_{i, j}^{u n F}=1$, and the stream is in vapor/gas phase, the pressure ratio in the horizontal direction is calculated by

$$
\begin{gathered}
\left(\frac{P_{i, j+1}}{P_{i, j}}\right)-P R^{u p}\left(2-z_{i, j}^{u n F}-z_{i, j}^{\text {phase }}\right) \leq P R_{i, j}^{F} \leq\left(\frac{P_{i, j+1}}{P_{i, j}}\right)+P R^{u p}\left(2-z_{i, j}^{\text {unF }}-z_{i, j}^{\text {phase }}\right), \\
\forall i, j, \\
1-\left(1-z_{i, j}^{\text {srF }}\right) \leq P R_{i, j}^{F} \leq 1+P R^{u p}\left(1-z_{i, j}^{\text {srF }}\right), \quad i \in \mathcal{I}, j \in \mathcal{J}, \\
1-z_{i, j}^{\text {phase }} \leq P R_{i, j}^{F} \leq 1+P R^{u p} z_{i, j}^{\text {phase }}, \quad i \in \mathcal{I}, j \in \mathcal{J} .
\end{gathered}
$$

For pressure change through a semi-restricted boundary, Eq. S90 sets the pressure ratio equal 
to 1, thus making the work terms zero. Similarly, Eq. S91 sets the pressure ratio for liquid streams equal to 1.

Similarly, we estimate the pressure ratio in vertical direction $\left(P R_{i, j}^{R}\right)$ by

$$
\begin{aligned}
& \left(\frac{P_{i+1, j}}{P_{i, j}}\right)-P R^{u p}\left(2-z_{i, j}^{u n R}-z_{i, j}^{\text {phase }}\right) \leq P R_{i, j}^{R} \leq\left(\frac{P_{i+1, j}}{P_{i, j}}\right)+P R^{u p}\left(2-z_{i, j}^{u n R}-z_{i, j}^{\text {phase }}\right), \forall i, j, \\
& 1-\left(1-z_{i, j}^{s r R}\right) \leq P R_{i, j}^{R} \leq 1+P R^{u p}\left(1-z_{i, j}^{s r R}\right), \quad i \in \mathcal{I}, j \in \mathcal{J} \\
& 1-z_{i, j}^{\text {phase }} \leq P R_{i, j}^{R} \leq 1+P R^{u p} z_{i, j}^{\text {phase }}, \quad i \in \mathcal{I}, j \in \mathcal{J} \text {. }
\end{aligned}
$$

Pressure ratio of the gas feed streams is determined from the ratio of the incoming block's pressure, $P_{i, j}$ to the pressure of the feed, $P_{f}^{\text {feed }}$ by

$$
P R_{i, j, f}^{\text {feed }}=\left(\frac{P_{i, j}}{P_{f}^{f e e d}}\right), \quad i \in \mathcal{I}, j \in \mathcal{J}, f \in \mathcal{G} \mathcal{F} \mathcal{S} .
$$

To set the pressure ratio of liquid feed streams to 1 we write,

$$
P R_{i, j, f}^{f e e d}=1, \quad i \in \mathcal{I}, j \in \mathcal{J}, f \in \mathcal{L} \mathcal{F} \mathcal{S}
$$

where, GFS and LFS are the sets of gas and liquid feed streams, respectively.

With this, the isentropic work term for horizontal streams, $F P_{i, j-1, k}$ and $F N_{i, j, k}$ are calculated by

$$
\begin{gathered}
\eta_{i s e} W_{i, j}^{\text {comp }, F P}-\frac{W_{i, j}^{e x p, F P}}{\eta_{i s e}}=\left(\sum_{k \in \mathcal{K}} F P_{i, j-1, k}\right) T_{i, j-1} R^{\text {gas }}\left(\frac{\gamma}{\gamma-1}\right)\left[\left(P R_{i, j-1}^{F}\right)^{\frac{(\gamma-1)}{\gamma}}-1\right] \\
i \in \mathcal{I}, j \in \mathcal{J}, \\
\eta_{i s e} W_{i, j}^{\text {comp }, F N}-\frac{W_{i, j}^{e x p, F N}}{\eta_{i s e}}=\left(\sum_{k \in \mathcal{K}} F N_{i, j, k}\right) T_{i, j+1} R^{\text {gas }}\left(\frac{\gamma}{\gamma-1}\right)\left[\left(\frac{1}{P R_{i, j}^{F}}\right)^{\frac{(\gamma-1)}{\gamma}}-1\right] \\
i \in \mathcal{I}, j \in \mathcal{J} .
\end{gathered}
$$

Similarly, for the vertical streams, $R P_{i-1, j, k}$ and $R N_{i, j, k}$, the isentropic work is calculated by

$$
\begin{gathered}
\eta_{i s e} W_{i, j}^{\text {comp }, R P}-\frac{W_{i, j}^{\text {exp }, R P}}{\eta_{\text {ise }}}=\left(\sum_{k \in \mathcal{K}} R P_{i-1, j, k}\right) T_{i-1, j} R^{\text {gas }}\left(\frac{\gamma}{\gamma-1}\right)\left[\left(P R_{i-1, j}^{R}\right)^{\frac{(\gamma-1)}{\gamma}}-1\right] \\
i \in \mathcal{I}, j \in \mathcal{J}, \\
\eta_{i s e} W_{i, j}^{\text {comp }, R N}-\frac{W_{i, j}^{\text {exp }, R N}}{\eta_{i s e}}=\left(\sum_{k \in \mathcal{K}} R N_{i, j, k}\right) T_{i+1, j} R^{\text {gas }}\left(\frac{\gamma}{\gamma-1}\right)\left[\left(\frac{1}{P R_{i, j}^{R}}\right)^{\frac{(\gamma-1)}{\gamma}}-1\right] \\
i \in \mathcal{I}, j \in \mathcal{J} .
\end{gathered}
$$


Additionally, work associated with the fresh feed streams and jump streams are calculated by

$$
\eta_{i s e} W_{i, j, f}^{c o m p, F S}-\frac{W_{i, j, f}^{e x p S}}{\eta_{i s e}}=\left(\sum_{k \in \mathcal{K}} M_{i, j, k, f}\right) T_{f}^{f e e d} R^{g a s}\left(\frac{\gamma}{\gamma-1}\right)\left[\left(P R_{i, j, f}^{f e e d}\right)^{\frac{(\gamma-1)}{\gamma}}-1\right]
$$

$$
i \in \mathcal{I}, j \in \mathcal{J}, f \in \mathcal{F S}
$$

$$
\eta_{i s e} W_{i, j, i^{\prime}, j^{\prime}}^{c o m p, J F}-\frac{W_{i, j, i^{\prime}, j^{\prime}}^{e x p, J F}}{\eta_{i s e}}=\left(\sum_{k \in \mathcal{K}} J_{i, j, i^{\prime}, j^{\prime}, k}\right) T_{i, j} R^{g a s}\left(\frac{\gamma}{\gamma-1}\right)\left[\left(\frac{P_{i^{\prime}, j^{\prime}}}{P_{i, j}}\right)^{\frac{(\gamma-1)}{\gamma}}-1\right]
$$

$$
i, j, i^{\prime}, j^{\prime} \in \mathcal{L} \mathcal{I} \mathcal{N} \mathcal{K} \text {. }
$$

The isentropic efficiency is considered to be $80 \%$ in both the case studies.

By using the above terms, energy balance (Eq. 15) can be rewritten as

$$
\begin{aligned}
& \sum_{k \in \mathcal{K}} E F P_{i, j-1, k}^{g}+\sum_{k \in \mathcal{K}} E F P_{i, j-1, k}^{l}-\sum_{k \in \mathcal{K}} E F N_{i, j-1, k}^{g}-\sum_{k \in \mathcal{K}} E F N_{i, j-1, k}^{l}+\sum_{k \in \mathcal{K}} E R P_{i-1, j, k}^{g} \\
& +\sum_{k \in \mathcal{K}} E R P_{i-1, j, k}^{l}-\sum_{k \in \mathcal{K}} E R N_{i-1, j, k}^{g}-\sum_{k \in \mathcal{K}} E R N_{i-1, j, k}^{l}-\sum_{k \in \mathcal{K}} E F P_{i, j, k}^{g}-\sum_{k \in \mathcal{K}} E F P_{i, j, k}^{l} \\
& +\sum_{k \in \mathcal{K}} E F N_{i, j, k}^{g}+\sum_{k \in \mathcal{K}} E F N_{i, j, k}^{l}-\sum_{k \in \mathcal{K}} E R P_{i, j, k}^{g}-\sum_{k \in \mathcal{K}} E R P_{i, j, k}^{l}+\sum_{k \in \mathcal{K}} E R N_{i, j, k}^{g} \\
& +\sum_{k \in \mathcal{K}} E R N_{i, j, k}^{l}+E G_{i, j}+\sum_{i^{\prime}, j^{\prime} \in \mathcal{L} \mathcal{I} \mathcal{N} \mathcal{K}} \sum_{k \in \mathcal{K}} E J_{i^{\prime}, j^{\prime}, i, j, k}^{g}+\sum_{i^{\prime}, j^{\prime} \in \mathcal{L} \mathcal{I} \mathcal{N} \mathcal{K}} \sum_{k \in \mathcal{K}} E J_{i^{\prime}, j^{\prime}, i, j, k}^{l} \\
& -\sum_{i^{\prime}, j^{\prime} \in \mathcal{L} \mathcal{I} \mathcal{N} \mathcal{K}} \sum_{k \in \mathcal{K}} E J_{i, j, i^{\prime}, j^{\prime}, k}^{g}-\sum_{i^{\prime}, j^{\prime} \in \mathcal{L} \mathcal{I} \mathcal{N} \mathcal{K}} \sum_{k \in \mathcal{K}} E J_{i, j, i^{\prime}, j^{\prime}, k}^{l}+E M_{i, j}-E N_{i, j}^{g}-E N_{i, j}^{l}+W_{i, j}^{c o m p, F P} \\
& +W_{i, j}^{c o m p, F N}+W_{i, j}^{c o m p, R P}+W_{i, j}^{c o m p, R N}+\sum_{f \in \mathcal{F} \mathcal{S}} W_{i, j, f s}^{c o m p, f}+\sum_{i^{\prime}, j^{\prime} \in \mathcal{L} \mathcal{I N \mathcal { K }}} W_{i, j, i^{\prime}, j^{\prime}}^{c o m p}-W_{i, j}^{e x p, F P} \\
& -W_{i, j}^{e x p, F N}-W_{i, j}^{\exp , R P}-W_{i, j}^{\exp , R N}-\sum_{f \in \mathcal{F} \mathcal{S}} W_{i, j, f \mathcal{S}}^{\exp , f}-\sum_{i^{\prime}, j^{\prime} \in \mathcal{L} \mathcal{I N \mathcal { K }}} W_{i, j, i^{\prime}, j^{\prime}}^{e x p, J F}+Q_{i, j}^{h}-Q_{i, j}^{c}=0, \\
& i \in \mathcal{I}, j \in \mathcal{J} \text {. }
\end{aligned}
$$

\section{Reaction Phenomena Model:}

The reaction phenomena model is presented in the main manuscript by Eq. 18, where, $R e_{i, j, k, r, c}$ is the amount of component $k$ consumed/generated by reaction $r$ on catalyst $c$ at block $B_{i, j}$. For different reaction model, $R e_{i, j, k, r, c}$ is determined by

$$
\begin{gathered}
R e_{i, j, k, r, c}=L R_{i, j, r, c} \gamma_{r, k} \operatorname{Conv}_{i, j, r, c}, \quad i \in \mathcal{I}, j \in \mathcal{J}, k \in \mathcal{K},(r, c) \in \mathcal{R} \mathcal{X C}, r \in \mathcal{S} \mathcal{T} \mathcal{O} \mathcal{C}, \\
R e_{i, j, k, r, c}=L R_{i, j, r, c} \gamma_{r, k} V_{i, j, c} \tau_{r}, \quad i \in \mathcal{I}, j \in \mathcal{J}, k \in \mathcal{K},(r, c) \in \mathcal{R} \mathcal{X C}, r \in \mathcal{K} \mathcal{I} \mathcal{N} \mathcal{C} \mathcal{T} \\
R e_{i, j, k, r, c}=L R_{i, j, r, c} \gamma_{r, k}, \quad i \in \mathcal{I}, j \in \mathcal{J}, k \in \mathcal{K},(r, c) \in \mathcal{R} \mathcal{X C}, r \in \mathcal{E} \mathcal{Q} \mathcal{I} \mathcal{L}
\end{gathered}
$$

Conv $v_{i, j, c, c}$ is a parameter that represents the fixed conversion for stoichiometric reaction models. In kinetic reaction model, $V_{i, j, c}$ and $\tau_{r}$ represents the catalyst amount, liquid holdup or reactor 
volume, and effectiveness factor for reaction $r$, respectively. The variable $L R_{i, j, r, c}$ is related to the binary variable $z_{i, j, c}^{r x n}$ by

$$
\begin{gathered}
L R_{i, j, r, c}=z_{i, j, c}^{r x n} L_{i, j, r}, \quad i \in \mathcal{I}, j \in \mathcal{J},(r, c) \in \mathcal{R} \mathcal{X C}, r \in \mathcal{K} \mathcal{I} \mathcal{N} \mathcal{E} \mathcal{T} \cup \mathcal{S} \mathcal{T O} \mathcal{I C} \\
L R_{i, j, r, c} \leq L R U_{i, j, r} z_{i, j, c}^{r x n}, \quad i \in \mathcal{I}, j \in \mathcal{J},(r, c) \in \mathcal{R} \mathcal{X C}, r \in \mathcal{E} \mathcal{Q U} \mathcal{I} \mathcal{L}
\end{gathered}
$$

The bilinear term $L R_{i, j, r, c}$ in Eq. S107, can be further linearized based on McCormick relaxations. Details of this linear reformulation can be found in Demirel et al. ${ }^{1}$

For stoichiometric reaction model, a key reactant $k^{*}$ is selected for every reaction $r$. Then to define the normalized amount of the key reactant $k^{*}$ entering into the block $B_{i, j}$ we write,

$$
L_{i, j, r}=-\frac{\phi_{i, j, k^{*}}}{\gamma_{r, k^{*}}}, \quad i \in \mathcal{I}, j \in \mathcal{J},\left(k^{*}, r\right) \in \mathcal{K} \mathcal{R}, r \in \mathcal{S} \mathcal{T O} \mathcal{I C},
$$

where, $\gamma_{r, k^{*}}$ is the stoichiometric coefficient of key reactant, $k^{*}$ in reaction $r . \phi_{i, j, k}$ denotes the total amount of component $k$ entering block $B_{i, j}$ and determined by

$$
\begin{aligned}
\phi_{i, j, k}= & F P_{i, j-1, k}+F N_{i, j, k}+R P_{i-1, j, k}+R N_{i, j, k}+\sum_{f \in \mathcal{F} \mathcal{S}} M_{i, j, k, f} \\
& +\sum_{\left(i^{\prime}, j^{\prime}\right) \in \mathcal{L} \mathcal{I N \mathcal { K }}} J_{i^{\prime}, j^{\prime}, i, j, k}, \quad i \in \mathcal{I}, j \in \mathcal{J}, k \in \mathcal{K} .
\end{aligned}
$$

For kinetic reaction model, reaction rate is expressed as

$$
L_{i, j, r}=k_{r}^{0} \exp \left(-\frac{E_{r}^{A}}{R T_{i, j}}\right) \prod_{k^{\prime} \in \mathcal{K} \mathcal{I} \mathcal{N}(k, r)} f\left(P_{i, j}, y_{i, j, k^{\prime}}\right), \quad i \in \mathcal{I}, j \in \mathcal{J}, r \in \mathcal{K} \mathcal{I} \mathcal{N} \mathcal{E} \mathcal{T}
$$

where, $k_{r}^{0}$ and $E_{r}^{A}$ represents the pre-exponential factor and the activation energy for reaction $r$, respectively. Function $f\left(P_{i, j}, y_{i, j, k^{\prime}}\right)$ determines the concentration dependence of the rate expression based on the set $\mathcal{K} \mathcal{I N}(k, r)$ that links the associated components $k_{1}, \ldots, k^{*}$ to the reaction $r$.

For equilibrium based model, the partial pressure $\left(P_{i, j} y_{i, j, k}\right)$ of the reaction mixture is determined from the equilibrium constant. Hence, we write,

$$
K e q_{i, j, r, c}\left(T_{i, j}\right)=\frac{\prod_{k \in \mathcal{P} \mathcal{R} \mathcal{O} \mathcal{D}_{k, r}}\left(P_{i, j} y_{i, j, k}\right)^{\gamma_{r, k}}}{\prod_{k \in \mathcal{R} \mathcal{E} \mathcal{A} \mathcal{C} \mathcal{T}_{k, r}}\left(P_{i, j} y_{i, j, k}\right)^{\gamma_{r, k}}}, \quad i \in \mathcal{I}, j \in \mathcal{J},(r, c) \in \mathcal{R} \mathcal{X C}, r \in \mathcal{E} \mathcal{Q} \mathcal{U} \mathcal{L},
$$

where, $K e q_{i, j, r, c}$ is the equilibrium constant for reaction $r$ on catalyst $c$ in block $B_{i, j}$, and $\mathcal{P} \mathcal{R O} \mathcal{D}_{k, r}$ and $\mathcal{R} \mathcal{E} \mathcal{A} \mathcal{C} \mathcal{T}_{k, r}$ are the set of products and reactants $k$ that participates in reaction $r$. In Eq. S108, $L R_{i, j, r, c}$ is a free variable when the corresponding equilibrium model is activated in block $B_{i, j}$ and the consumption/generation of component is estimated by material balances with reaction stoichiometry (Eq. S106) and block compositions (Eq. S112). 
Depending on the reactions and catalyst involved, the temperature and pressure of the reactor blocks are restricted within a certain operating range. Therefore, we impose following logical constraints,

$$
\begin{aligned}
& T_{i, j} \leq T_{c}^{\text {max }}+\left(T^{\text {max }}-T_{c}^{\text {max }}\right)\left(1-z_{i, j, c}^{r x n}\right), \quad i \in \mathcal{I}, j \in \mathcal{J}, c \in \mathcal{C} \mathcal{A} \mathcal{T}, \\
& T_{i, j} \geq T_{c}^{\text {min }}-\left(T_{c}^{\text {min }}-T^{\text {min }}\right)\left(1-z_{i, j, c}^{r x n}\right), \quad i \in \mathcal{I}, j \in \mathcal{J}, c \in \mathcal{C} \mathcal{A} \mathcal{T}, \\
& P_{i, j} \leq P_{c}^{\text {max }}+\left(P^{\text {max }}-P_{c}^{\text {max }}\right)\left(1-z_{i, j, c}^{r x n}\right), \quad i \in \mathcal{I}, j \in \mathcal{J}, c \in \mathcal{C} \mathcal{A} \mathcal{T}, \\
& P_{i, j} \geq P_{c}^{\text {min }}-\left(P_{c}^{\text {min }}-P^{\text {min }}\right)\left(1-z_{i, j, c}^{r x n}\right), \quad i \in \mathcal{I}, j \in \mathcal{J}, c \in \mathcal{C} \mathcal{A} \mathcal{T} .
\end{aligned}
$$

Here, $T_{c}^{\max }$ and $T_{c}^{\text {min }}$ are the maximum and minimum allowable temperature of catalyst $c$, respectively. Similarly, $P_{c}^{\max }$ and $P_{c}^{\min }$ are the maximum and minimum allowable pressure for catalyst $c$, respectively. If catalyst $c$ is present in reactor block $B_{i, j}$, then $z_{i, j, c}^{r x n}=1$ and the block temperature $\left(T_{i, j}\right)$ and pressure $\left(P_{i, j}\right)$ are bounded. Otherwise, block temperature and pressure are free and can vary within the maximum and minimum temperature ( $T^{\text {max }}$ and $T^{\text {min }}$, respectively) and pressure $\left(P^{\max }\right.$ and $P^{\min }$, respectively) of the superstructure.

\section{Separation Phenomena Model:}

According to Eq. 20 in the main manuscript, when a semi-restricted boundary is occupied with phenomena $s$ and membrane material $m$, then the rate of mass transfer through the membrane boundary is determined by the mass transfer coefficient, chemical potential difference and surface area of the membrane. Therefore, to determine the flow rate of component $k$ in the horizontal direction through a membrane boundary, we write,

$$
\begin{aligned}
F_{i, j, k} \geq & \lambda_{i, j, k, s, m} \times\left(\mu_{i, j, k, s, m}-\mu_{i, j+1, k, s, m}\right) \times A_{i, j, s, m}^{F} \\
& -M_{i, j, k, s, m}^{\text {rateF }} \times\left(1-z_{i, j, s, m}^{F}\right), \quad i \in \mathcal{I}, j \in \mathcal{J}, k \in \mathcal{K},(s, m) \in \mathcal{S} \mathcal{M}, \\
F_{i, j, k} \leq & \lambda_{i, j, k, s, m} \times\left(\mu_{i, j, k, s, m}-\mu_{i, j+1, k, s, m}\right) \times A_{i, j, s, m}^{F} \\
& +M_{i, j, k, s, m}^{\text {rateF }} \times\left(1-z_{i, j, s, m}^{F}\right), \quad i \in \mathcal{I}, j \in \mathcal{J}, k \in \mathcal{K},(s, m) \in \mathcal{S} \mathcal{M},
\end{aligned}
$$

where, $\lambda_{i, j, k, s, m}$ is the mass transfer coefficient of component $k$ for separation phenomena $s$ and membrane $m, \mu_{i, j, k, s, m}$ is the chemical potential of component $k$ at block $B_{i, j}$ for membrane $m$ with phenomena $s$, and $A_{i, j, s, m}^{F}$ is the surface area of membrane material $m$ at block $B_{i, j}$ occupying the common boundary between block $B_{i, j}$ and $B_{i, j+1}$.

Similarly, for the vertical direction, we have,

$$
\begin{aligned}
R_{i, j, k} \geq & \lambda_{i, j, k, s, m} \times\left(\mu_{i, j, k, s, m}-\mu_{i+1, j, k, s, m}\right) \times A_{i, j, s, m}^{R} \\
& -M_{i, j, k, s, m}^{\text {rateR }} \times\left(1-z_{i, j, s, m}^{R}\right), \quad i \in \mathcal{I}, j \in \mathcal{J}, k \in \mathcal{K},(s, m) \in \mathcal{S} \mathcal{M}, \\
R_{i, j, k} \leq & \lambda_{i, j, k, s, m} \times\left(\mu_{i, j, k, s, m}-\mu_{i+1, j, k, s, m}\right) \times A_{i, j, s, m}^{R} \\
& +M_{i, j, k, s, m}^{\text {rateR }} \times\left(1-z_{i, j, s, m}^{R}\right), \quad i \in \mathcal{I}, j \in \mathcal{J}, k \in \mathcal{K},(s, m) \in \mathcal{S} \mathcal{M} .
\end{aligned}
$$


Mass transfer coefficients can be estimated from diffusion coefficients and membrane thickness or can be considered as a lumped parameter such as permeance. Furthermore, it can be considered as a variable to study the performance of membrane-based reactive separation process for finding optimal membrane properties.

Depending on the permeation phenomena and mixing assumption, the description of the chemical potential can be different. For instance, when the permeation phenomena is gas $(G P)$ and/or vapor permeation $(V P)$, with the ideal gas assumption and the complete mixing assumption in both side of the membrane, chemical potential can be defined as partial pressure,

$$
\mu_{i, j, k, s, m}=P_{i, j} \times y_{i, j, k}, \quad i \in \mathcal{I}, j \in \mathcal{J}, k \in \mathcal{K},(s, m) \in \mathcal{S M}, s \in\{G P, V P\} .
$$

When chemical potential terms differ between permeate and retentate side, we assign separate terms for permeate and retentate side chemical potential as $\mu_{i, j, k, s, m}^{p e r}$ and $\mu_{i, j, k, s, m}^{r e t}$. For instance, for pervaporation $(P V)$, the phase of the permeate side is vapor while the phase of retentate side is liquid. So, for permeate side, similar relationship as $G P$ and $V P$ can be used. However, for the retentate side, vapor pressure $\left(P_{i, j, k}^{s a t}\right)$ and liquid activity coefficient $\left(\Gamma_{i, j, k, s, m}\right)$ is used to determine chemical potential,

$$
\mu_{i, j, k, s, m}^{r e t}=P_{i, j, k}^{s a t} \times y_{i, j, k} \times \Gamma_{i, j, k, s, m}, \quad i \in \mathcal{I}, j \in \mathcal{J}, k \in \mathcal{K},(s, m) \in \mathcal{S} \mathcal{M}, s \in\{P V\} .
$$

The direction of flow through the semi-restricted boundary determines the permeate and retentate side of the membrane. However, when we define chemical potential for permeate and retentate side with different terms, the mass transfer constraints described by Eqs. S115-S118 is rewritten as

$$
\begin{aligned}
F P_{i, j, k} \geq & \lambda_{i, j, k, s, m} \times\left(\mu_{i, j, k, s, m}^{r e t}-\mu_{i, j+1, k, s, m}^{\text {per }}\right) \times A_{i, j, s, m}^{F} \\
& -M_{i, j, k, s, m}^{\text {rateF }} \times\left(2-z_{i, j, s, m}^{F}-z_{i, j}^{F p l u s}\right), \quad i \in \mathcal{I}, j \in \mathcal{J}, k \in \mathcal{K},(s, m) \in \mathcal{S M}, \\
F P_{i, j, k} \leq & \lambda_{i, j, k, s, m} \times\left(\mu_{i, j, k, s, m}^{r e t}-\mu_{i, j+1, k, s, m}^{\text {per }}\right) \times A_{i, j, s, m}^{F} \\
& +M_{i, j, k, s, m}^{\text {rateF }} \times\left(2-z_{i, j, s, m}^{F}-z_{i, j}^{F p l u s}\right), \quad i \in \mathcal{I}, j \in \mathcal{J}, k \in \mathcal{K},(s, m) \in \mathcal{S} \mathcal{M}, \\
F N_{i, j, k} \geq & \lambda_{i, j, k, s, m} \times\left(\mu_{i, j+1, k, s, m}^{r e t}-\mu_{i, j, k, s, m}^{\text {per }}\right) \times A_{i, j, s, m}^{F} \\
& -M_{i, j, k, s, m}^{\text {rateF }} \times\left(1-z_{i, j, s, m}^{F}+z_{i, j}^{F p l u s}\right), \quad i \in \mathcal{I}, j \in \mathcal{J}, k \in \mathcal{K},(s, m) \in \mathcal{S} \mathcal{M}, \\
F N_{i, j, k} \leq & \lambda_{i, j, k, s, m} \times\left(\mu_{i, j+1, k, s, m}^{r e t}-\mu_{i, j, k, s, m}^{\text {per }}\right) \times A_{i, j, s, m}^{F} \\
& +M_{i, j, k, s, m}^{\text {rateF }} \times\left(1-z_{i, j, s, m}^{F}+z_{i, j}^{F p l u s}\right), \quad i \in \mathcal{I}, j \in \mathcal{J}, k \in \mathcal{K},(s, m) \in \mathcal{S M}, \\
& \quad M_{i, j, k, s, m}^{\text {rateR }} \times\left(2-z_{i, j, s, m}^{R}-z_{i, j}^{\text {Rplus }}\right), \quad i \in \mathcal{I}, j \in \mathcal{J}, k \in \mathcal{K},(s, m) \in \mathcal{S} \mathcal{M},
\end{aligned}
$$




$$
\begin{aligned}
R P_{i, j, k} \leq & \lambda_{i, j, k, s, m} \times\left(\mu_{i, j, k, s, m}^{r e t}-\mu_{i+1, j, k, s, m}^{\text {per }}\right) \times A_{i, j, s, m}^{R} \\
& +M_{i, j, k, s, m}^{\text {rateR }} \times\left(2-z_{i, j, s, m}^{R}-z_{i, j}^{\text {Rplus }}\right), \quad i \in \mathcal{I}, j \in \mathcal{J}, k \in \mathcal{K},(s, m) \in \mathcal{S M}, \\
R N_{i, j, k} \geq & \lambda_{i, j, k, s, m} \times\left(\mu_{i+1, j, k, s, m}^{\text {ret }}-\mu_{i, j, k, s, m}^{\text {per }}\right) \times A_{i, j, s, m}^{R} \\
& -M_{i, j, k, s, m}^{\text {rateR }} \times\left(1-z_{i, j, s, m}^{R}+z_{i, j}^{\text {Rplus }}\right), \quad i \in \mathcal{I}, j \in \mathcal{J}, k \in \mathcal{K},(s, m) \in \mathcal{S} \mathcal{M}, \\
R N_{i, j, k} \leq & \lambda_{i, j, k, s, m} \times\left(\mu_{i+1, j, k, s, m}^{\text {ret }}-\mu_{i, j, k, s, m}^{\text {per }}\right) \times A_{i, j, s, m}^{R} \\
& +M_{i, j, k, s, m}^{\text {rateR }} \times\left(1-z_{i, j, s, m}^{R}+z_{i, j}^{\text {Rplus }}\right), \quad i \in \mathcal{I}, j \in \mathcal{J}, k \in \mathcal{K},(s, m) \in \mathcal{S} \mathcal{M} .
\end{aligned}
$$

Eqs. S121-S122 are the constraints for horizontal direction when the block in the left is assigned as the retentate side and the block in the right is assigned as the permeate side. Conversely, Eqs. S123-S124 holds true when permeate side is assigned to the left hand side block and retentate side is on the right hand side block. Similarly, Eqs. S125-S128 represents the constraints for flow in the vertical direction.

If apart from the flow through membrane boundary, there exist no other outlet stream such as flow through the unrestricted interblock boundaries, product flow or jump flow from a block (i.e. if Eqs. S5-S12 and Eqs. 12-13 in the main manuscript does not hold true), then the block composition becomes arbitrary. In this case, flow rate through the membrane does not depend upon driving force constraints and the membrane boundary can act as an ideal separator i.e. the permeate flow rates becomes equal to the block inlet flow rates. To prevent this, we define a parameter $\theta^{\max }<1$. Here, $\theta^{\max }$ serves as the maximum stage-cut for the membrane boundary. For membrane separation, stage-cut is defined as the ratio of permeate and feed flow rates. Apart from the flow through the membrane boundary, to ensure at least one outlet flow through interblock, product or jump streams, we write,

$$
\begin{gathered}
\left(1-\theta^{\max }\right) \sum_{k \in \mathcal{K}} \phi_{i, j, k} \leq F P_{i, j}^{u n}+R P_{i, j}^{u n}+F N_{i, j-1}^{u n}+R N_{i-1, j}^{u n}+\sum_{k \in \mathcal{K}} \sum_{p \in \mathcal{P} \mathcal{S}} N_{i, j, k, p} \\
+\sum_{i^{\prime}, j^{\prime} \in \mathcal{L} \mathcal{I} \mathcal{N} \mathcal{K}} \sum_{k \in \mathcal{K}} J_{i, j, i^{\prime}, j^{\prime}, k}, \quad i \in \mathcal{I}, j \in \mathcal{J}, \\
F P_{i, j}^{u n} \leq F U z_{i, j}^{u n F} ; \quad F P_{i, j}^{u n} \geq \sum_{k \in \mathcal{K}} F P_{i, j, k}-F U\left(1-z_{i, j}^{u n F}\right) ; \quad F P_{i, j}^{u n} \leq \sum_{k \in \mathcal{K}} F P_{i, j, k}, \quad \forall i, j, \\
F N_{i, j}^{u n} \leq F U z_{i, j}^{u n F} ; \quad F N_{i, j}^{u n} \geq \sum_{k \in \mathcal{K}} F N_{i, j, k}-F U\left(1-z_{i, j}^{u n F}\right) ; \quad F N_{i, j}^{u n} \leq \sum_{k \in \mathcal{K}} F N_{i, j, k}, \quad \forall i, j, \\
R P_{i, j}^{u n} \leq R U z_{i, j}^{u n R} ; \quad R P_{i, j}^{u n} \geq \sum_{k \in \mathcal{K}} R P_{i, j, k}-R U\left(1-z_{i, j}^{u n R}\right) ; \quad R P_{i, j}^{u n} \leq \sum_{k \in \mathcal{K}} R P_{i, j, k} \quad \forall i, j, \\
R N_{i, j}^{u n} \leq R U z_{i, j}^{u n R} ; \quad R N_{i, j}^{u n} \geq \sum_{k \in \mathcal{K}} R N_{i, j, k}-R U\left(1-z_{i, j}^{u n R}\right) ; \quad R P_{i, j}^{u n} \leq \sum_{k \in \mathcal{K}} R P_{i, j, k} \quad \forall i, j .
\end{gathered}
$$

According to Eq. S129, a certain fraction of the total inlet flow $\sum_{k \in \mathcal{K}} \phi_{i, j, k}$ has to leave the block 
through either interblock, product, or jump streams. Eqs. S130-S133 estimates the total flow rates of interblock streams through the corresponding unrestricted boundary.

\section{Task Specific Constraints:}

Eq. 21 in the main manuscript represents the set of equipment specific constraints. For instance, if multiple reactor blocks are used in series to represent a plug flow reactor (PFR), then to ensure isobaric reaction condition we impose,

$$
\begin{array}{ll}
P_{i, j} \leq P_{i, j+1}+\left(P^{\text {max }}-P^{\text {min }}\right)\left(2-z_{i, j, c}^{r x n}-z_{i, j+1, c}^{r x n}\right), & i \in \mathcal{I}, j \in \mathcal{J}, c \in \mathcal{C} \mathcal{A} \mathcal{T}, \\
P_{i, j} \geq P_{i, j+1}-\left(P^{\text {max }}-P^{\text {min }}\right)\left(2-z_{i, j, c}^{r x n}-z_{i, j+1, c}^{r x n}\right), & i \in \mathcal{I}, j \in \mathcal{J}, c \in \mathcal{C} \mathcal{A} \mathcal{T}, \\
P_{i, j} \leq P_{i+1, j}+\left(P^{\max }-P^{\text {min }}\right)\left(2-z_{i, j, c}^{r x n}-z_{i+1, j, c}^{r x n}\right), & i \in \mathcal{I}, j \in \mathcal{J}, c \in \mathcal{C} \mathcal{A} \mathcal{T}, \\
P_{i, j} \geq P_{i+1, j}-\left(P^{\max }-P^{\text {min }}\right)\left(2-z_{i, j, c}^{r x n}-z_{i+1, j, c}^{r x n}\right), & i \in \mathcal{I}, j \in \mathcal{J}, c \in \mathcal{C} \mathcal{A} \mathcal{T} .
\end{array}
$$

Eq. S134 states that, if catalyst $c$ is present in two adjacent horizontal blocks, $B_{i, j}$ and $B_{i, j+1}$, then the pressure of those blocks become equal. Eq. S135, imposed the same constraint for blocks in vertical direction.

Similarly when multiple blocks are used to represent PFR, the catalyst amount, $V_{i, j, c}$ has to be equal in all the reactor blocks. Therefore, we write,

$$
\begin{array}{ll}
V_{i, j, c} \leq V_{i, j+1, c}+V_{c}^{\max }\left(2-z_{i, j, c}^{r x n}-z_{i, j+1, c}^{r x n}\right), & i \in \mathcal{I}, j \in \mathcal{J}, c \in \mathcal{C} \mathcal{A} \mathcal{T}, \\
V_{i, j, c} \geq V_{i, j+1, c}-V_{c}^{\max }\left(2-z_{i, j, c}^{r x n}-z_{i, j+1, c}^{r x n}\right), & i \in \mathcal{I}, j \in \mathcal{J}, c \in \mathcal{C} \mathcal{A} \mathcal{T}, \\
V_{i, j, c} \leq V_{i+1, j, c}+V_{c}^{\max }\left(2-z_{i, j, c}^{r x n}-z_{i+1, j, c}^{r x n}\right), & i \in \mathcal{I}, j \in \mathcal{J}, c \in \mathcal{C} \mathcal{A} \mathcal{T}, \\
V_{i, j, c} \geq V_{i+1, j, c}-V_{c}^{\max }\left(2-z_{i, j, c}^{r x n}-z_{i+1, j, c}^{r x n}\right), & i \in \mathcal{I}, j \in \mathcal{J}, c \in \mathcal{C} \mathcal{A} \mathcal{T} .
\end{array}
$$

In most problems, variable $V_{i, j, c}$ remains as a free variable bounded by $V_{c}^{\max }$. However, it can also be determined from the reactor geometry such as reactor tube's diameter, $D_{t u b e}$; number, $N_{\text {tube }}$; reactor length, $L_{\text {tube }}$; catalyst packing density, $\rho_{c}$; and reactor bed voidage fraction, $\varepsilon$ by

$$
V_{i, j, c}=\frac{\frac{\pi}{4} D_{\text {tube }}^{2} L_{\text {tube }} \rho_{c} N_{\text {tube }}(1-\varepsilon)}{N_{R B}}, \quad i, j \in \mathcal{R} \mathcal{X} \mathcal{N B}, c \in \mathcal{C} \mathcal{A} \mathcal{T}
$$

Here, $N_{R B}$ is the number of blocks used to represent the reactor. If $N_{R B}=1$, then all the catalyst is packed within a single block and it represents a CSTR. On the other hand, for a PFR, $N_{R B}>1$ and catalyst is evenly distributed among all the reactor blocks. However, to ensure $N_{R B}$ does not become zero, we pre-specify the reaction blocks within the superstructure by the set $\mathcal{R} \mathcal{X N B}$ and write,

$$
\sum_{i, j \in \mathcal{R} \mathcal{X} \mathcal{N B}} z_{i, j, c}^{r x n} \geq 1, \quad c \in \mathcal{C} \mathcal{A} \mathcal{T}
$$

Similarly, the membrane area can be determined from the reactor geometry by 


$$
\begin{aligned}
& A_{i, j, s, m}^{F}=\frac{\pi D_{\text {tube }} L_{\text {tube }} N_{\text {tube }}}{N_{R B}}, \quad i, j \in \mathcal{R} \mathcal{X N \mathcal { N }},(s, m) \in \mathcal{S} \mathcal{M}, \\
& A_{i, j, s, m}^{R}=\frac{\pi D_{\text {tube }} L_{\text {tube }} N_{\text {tube }}}{N_{R B}}, \quad i, j \in \mathcal{R} \mathcal{X N \mathcal { N }},(s, m) \in \mathcal{S} \mathcal{M} .
\end{aligned}
$$

Generally, membrane materials cannot withstand higher temperatures and operate below a certain maximum temperature. Therefore, when a membrane $m$ is present between two blocks then the temperature of these two blocks cannot exceed the maximum allowable temperature $T_{m}^{\max }$ of the membrane. To impose these constraints we write,

$$
\begin{gathered}
T_{i, j} \leq T_{m}^{\text {max }}+\left(T^{\text {max }}-T^{\text {min }}\right)\left(1-z_{i, j, s, m}^{s F}\right), \quad i \in \mathcal{I}, j \in \mathcal{J},(s, m) \in \mathcal{S M}, \\
T_{i, j} \leq T_{m}^{\text {max }}+\left(T^{\text {max }}-T^{\text {min }}\right)\left(1-z_{i, j, s, m}^{s R}\right), \quad i \in \mathcal{I}, j \in \mathcal{J},(s, m) \in \mathcal{S M}, \\
T_{i, j+1} \leq T_{m}^{\text {max }}+\left(T^{\text {max }}-T^{\text {min }}\right)\left(1-z_{i, j, s, m}^{s F}\right), \quad i \in \mathcal{I}, j \in \mathcal{J},(s, m) \in \mathcal{S M}, \\
T_{i+1, j} \leq T_{m}^{\text {max }}+\left(T^{\text {max }}-T^{\text {min }}\right)\left(1-z_{i, j, s, m}^{s R}\right), \quad i \in \mathcal{I}, j \in \mathcal{J},(s, m) \in \mathcal{S M} .
\end{gathered}
$$

Apart from the basic separation model described above, some additional constrains are also considered to represent a more realistic membrane operation. For instance, when a membrane reactor is represented by a series of multiple building blocks (PFR), then the membrane area in each of the blocks has to be equal. This is imposed by the following constraints:

$$
\begin{array}{ll}
A_{i, j, s, m}^{F} \leq A_{i+1, j, s, m}^{F}+A_{m}^{\max }\left(2-z_{i, j, s, m}^{s F}-z_{i+1, j, s, m}^{s F}\right), & i \in \mathcal{I}, j \in \mathcal{J},(s, m) \in \mathcal{S} \mathcal{M}, \\
A_{i, j, s, m}^{F} \geq A_{i+1, j, s, m}^{F}-A_{m}^{\max }\left(2-z_{i, j, s, m}^{s F}-z_{i+1, j, s, m}^{s F}\right), & i \in \mathcal{I}, j \in \mathcal{J},(s, m) \in \mathcal{S} \mathcal{M}, \\
A_{i, j, s, m}^{R} \leq A_{i, j+1, s, m}^{R}+A_{m}^{\max }\left(2-z_{i, j, s, m}^{s R}-z_{i, j+1, s, m}^{s R}\right), & i \in \mathcal{I}, j \in \mathcal{J},(s, m) \in \mathcal{S} \mathcal{M}, \\
A_{i, j, s, m}^{R} \geq A_{i, j+1, s, m}^{R}-A_{m}^{\max }\left(2-z_{i, j, s, m}^{s R}-z_{i, j+1, s, m}^{s R}\right), & i \in \mathcal{I}, j \in \mathcal{J},(s, m) \in \mathcal{S} \mathcal{M} .
\end{array}
$$

\section{S2 Reaction Kinetics}

In this section, we provide the reaction kinetic models used in the case studies.

Methanol Synthesis Reaction: In the first case study, the kinetics model presented by Bussche and Froment ${ }^{2}$ is considered for methanol synthesis reactions. In this model, only $\mathrm{CO}_{2}$ hydrogenation reaction (Eq. 29) and reverse water gas shift reaction (Eq. 30) are considered. The reaction rate expressions are presented by

$$
\begin{gathered}
r_{\mathrm{CH}_{3} \mathrm{OH}}=\frac{k_{1}\left(P_{\mathrm{H}_{2}} P_{\mathrm{CO}_{2}}-\frac{P_{\mathrm{H}_{2} \mathrm{O}} P_{\mathrm{CH}_{3} \mathrm{OH}}}{K_{1} P_{\mathrm{H}_{2}}^{2}}\right)}{\left(1+k_{\mathrm{H}_{2}} \sqrt{P_{\mathrm{H}_{2}}}+k_{\mathrm{H}_{2} \mathrm{O}} P_{\mathrm{H}_{2} \mathrm{O}}+k_{\mathrm{H}_{2} \mathrm{O} / \mathrm{H}_{2}} \frac{P_{\mathrm{H}_{2} \mathrm{O}}}{P_{\mathrm{H}_{2}}}\right)^{3}}, \\
r_{\mathrm{RWGS}}=\frac{k_{2}\left(P_{\mathrm{CO}_{2}}-\frac{K_{2} P_{\mathrm{H}_{2} \mathrm{O}} P_{\mathrm{CO}}}{P_{\mathrm{H}_{2}}}\right)}{\left(1+k_{\mathrm{H}_{2}} \sqrt{P_{\mathrm{H}_{2}}}+k_{\mathrm{H}_{2} \mathrm{O}} P_{\mathrm{H}_{2} \mathrm{O}}+k_{\mathrm{H}_{2} \mathrm{O} / \mathrm{H}_{2}} \frac{P_{\mathrm{H}_{2} \mathrm{O}}}{P_{\mathrm{H}_{2}}}\right)} .
\end{gathered}
$$


The corresponding rate, adsorption equilibrium, and equilibrium constants are presented in Table S1-S2.

Table S1: Reaction rate and adsorption equilibrium constants for methanol synthesis reactions.

\begin{tabular}{cccc}
\hline Constants & Units & $\mathrm{A}$ & $\mathrm{B}\left({\left.\mathrm{kJ} . \mathrm{mol}^{-1}\right)}^{-1}\right.$ \\
\hline$k_{1}$ & $\mathrm{~mol}_{\mathrm{kg}}^{\mathrm{cat}}-\mathrm{s}^{-1} \cdot \mathrm{bar}^{-2}$ & 1.07 & 36.70 \\
$k_{2}$ & $\mathrm{~mol}_{\mathrm{kg}}^{-1} \cdot \mathrm{cat}^{-1} \cdot \mathrm{bar}^{-1}$ & $1.22 \times 10^{10}$ & -94.76 \\
$k_{\mathrm{H}_{2}}$ & $\mathrm{bar}^{-0.5}$ & 0.499 & 17.20 \\
$k_{\mathrm{H}_{2} \mathrm{O}}$ & $\mathrm{bar}^{-1}$ & $6.62 \times 10^{-11}$ & 124.12 \\
$k_{\mathrm{H}_{2} \mathrm{O} / \mathrm{H}_{2}}$ & - & 3453.38 & 0 \\
$k=\mathrm{A} \exp \left(\frac{\mathrm{B}}{\mathrm{RT}}\right)$ & & & \\
\hline
\end{tabular}

Table S2: Reaction equilibrium constants for methanol synthesis reactions.

\begin{tabular}{ccc}
\hline Constants & Units & Expression \\
\hline$K_{1}$ & bar $^{-2}$ & $\exp \left(\frac{3066}{\mathrm{~T}}-10.592\right)$ \\
$K_{2}$ & - & $\exp \left(\frac{2073}{\mathrm{~T}}-2.029\right)$ \\
\hline
\end{tabular}

Partial Oxidation of Methane (POM) Reaction: In the second case study, the reaction kinetics model proposed by De Groote and Froment ${ }^{3}$ is considered for methane partial oxidation reactions. The reaction rate expression for total oxidation of methane (Eq. 31) is proposed by Trimm and Lam. ${ }^{4}$ However, their model is based on Pt supported catalyst. For this reason, the modified rate expression proposed De Smet et al. ${ }^{5}$ has been considered, which is better suited for $\mathrm{Ni}$ based catalyst. The Langmuir-Hinshelwood type rate equation is presented by

$$
r_{1}=\frac{k_{11} P_{\mathrm{CH}_{4}} P_{\mathrm{O}_{2}}}{\left(1+K_{\mathrm{O}_{2}}^{0} P_{\mathrm{O}_{2}}+K_{\mathrm{CH}_{4}}^{0} P_{\mathrm{CH}_{4}}\right)^{2}}+\frac{k_{12} P_{\mathrm{CH}_{4}} P_{\mathrm{O}_{2}}}{\left(1+K_{\mathrm{O}_{2}}^{0} P_{\mathrm{O}_{2}}+K_{\mathrm{CH}_{4}}^{0} P_{\mathrm{CH}_{4}}\right)} .
$$

The rate expression for the SMR reactions (Eq. 32 and Eq. 34) and water gas shift reaction (Eq. 33) are proposed by $\mathrm{Xu}$ and Froment. ${ }^{6}$ The rate expression are presented by

$$
\begin{aligned}
& r_{2}=\frac{\frac{k_{2}}{P_{\mathrm{H}_{2}}^{2.5}}\left(P_{\mathrm{CH}_{4}} P_{\mathrm{H}_{2} \mathrm{O}}-\frac{P_{\mathrm{H}_{2}}^{3} P_{\mathrm{CO}}}{K_{\mathrm{eq} 2}}\right)}{\left(1+K_{\mathrm{CO}} P_{\mathrm{CO}}+K_{\mathrm{H}_{2}} P_{\mathrm{H}_{2}}+K_{\mathrm{CH}_{4}} P_{\mathrm{CH}_{4}}+K_{\mathrm{H}_{2} \mathrm{O}} \frac{P_{\mathrm{H}_{2} \mathrm{O}}}{P_{\mathrm{H}_{2}}}\right)^{2}}, \\
& r_{3}=\frac{\frac{k_{3}}{P_{\mathrm{H}_{2}}}\left(P_{\mathrm{CO}} P_{\mathrm{H}_{2} \mathrm{O}}-\frac{P_{\mathrm{H}_{2}} P_{\mathrm{CO}_{2}}}{K_{\mathrm{eq}^{3}}}\right)}{\left(1+K_{\mathrm{CO}} P_{\mathrm{CO}}+K_{\mathrm{H}_{2}} P_{\mathrm{H}_{2}}+K_{\mathrm{CH}_{4}} P_{\mathrm{CH}_{4}}+K_{\mathrm{H}_{2} \mathrm{O}} \frac{P_{\mathrm{H}_{2} \mathrm{O}}}{P_{\mathrm{H}_{2}}}\right)^{2}},
\end{aligned}
$$




$$
r_{4}=\frac{\frac{k_{4}}{P_{\mathrm{H}_{2}}^{3.5}}\left(P_{\mathrm{CH}_{4}} P_{\mathrm{H}_{2} \mathrm{O}}^{2}-\frac{P_{\mathrm{H}_{2}}^{4} P_{\mathrm{CO}_{2}}}{K_{\mathrm{eq} 4}}\right)}{\left(1+K_{\mathrm{CO}} P_{\mathrm{CO}}+K_{\mathrm{H}_{2}} P_{\mathrm{H}_{2}}+K_{\mathrm{CH}_{4}} P_{\mathrm{CH}_{4}}+K_{\mathrm{H}_{2} \mathrm{O}} \frac{P_{\mathrm{H}_{2} \mathrm{O}}}{P_{\mathrm{H}_{2}}}\right)^{2}} .
$$

Kinetic parameters for all four reactions are tabulated in Table S3-S5. In Table S3, $\eta$ represents the effectiveness factors of reactions.

Table S3: Arrhenius parameters and effectiveness factors for POM reactions.

\begin{tabular}{ccccc}
\hline Reactions & Constants & $\mathrm{A}\left(\mathrm{mol}_{\mathrm{kg}}^{\mathrm{cat}}-\mathrm{s}^{-1}\right)$ & $\mathrm{E}_{\mathrm{a}}\left(\mathrm{kJ} . \mathrm{mol}^{-1}\right)$ & $\eta$ \\
\hline 1 & $k_{11}$ & $8.11 \times 10^{5} \mathrm{bar}^{-2}$ & 86.0 & 0.05 \\
2 & $k_{12}$ & $8.82 \times 10^{5} \mathrm{bar}^{-2}$ & 86.0 & \\
3 & $k_{2}$ & $1.17 \times 10^{15} \mathrm{bar}^{0.5}$ & 240.0 & 0.07 \\
4 & $k_{3}$ & $5.43 \times 10^{5} \mathrm{bar}^{-1}$ & 67.1 & 0.70 \\
$k=\mathrm{A} \exp \left(\frac{-\mathrm{E}_{\mathrm{a}}}{\mathrm{RT}}\right)$ & $k_{4}$ & $2.83 \times 10^{14} \mathrm{bar}^{0.5}$ & 243.0 & 0.06 \\
\hline
\end{tabular}

Table S4: Van't Hoff parameters for the POM adsorption reactions.

\begin{tabular}{ccc}
\hline Constants & $\mathrm{A}$ & $\Delta \mathrm{H}^{0}\left(\mathrm{~kJ} . \mathrm{mol}^{-1}\right)$ \\
\hline$K_{\mathrm{CH}_{4}}^{0}$ & $1.26 \times 10^{-1} \mathrm{bar}^{-1}$ & -27.3 \\
$K_{\mathrm{O}_{2}}$ & $7.87 \times 10^{-7} \mathrm{bar}^{-1}$ & -92.8 \\
$K_{\mathrm{CO}}$ & $8.23 \times 10^{-5} \mathrm{bar}^{-1}$ & -70.7 \\
$K_{\mathrm{H}_{2}}$ & $6.62 \times 10^{-9} \mathrm{bar}^{-1}$ & -82.9 \\
$K_{\mathrm{CH}_{4}}$ & $6.65 \times 10^{-4} \mathrm{bar}^{-1}$ & -38.3 \\
$K_{\mathrm{H}_{2} \mathrm{O}}$ & $1.77 \times 10^{5}$ & 88.7 \\
$K=\mathrm{A} \exp \left(\frac{-\Delta \mathrm{H}^{0}}{\mathrm{RT}}\right)$ & & \\
\hline
\end{tabular}

Table S5: Reaction equilibrium constants for POM reactions.

\begin{tabular}{ccc}
\hline Constants & Units & Expression \\
\hline$K_{\text {eq2 }}$ & bar $^{2}$ & $\exp \left(\frac{-26830}{\mathrm{~T}}+30.114\right)$ \\
$K_{\text {eq3 }}$ & - & $\exp \left(\frac{4400}{\mathrm{~T}}-4.036\right)$ \\
$K_{\text {eq4 }}$ & bar $^{2}$ & $\exp \left(\frac{-22430}{\mathrm{~T}}+26.078\right)$ \\
\hline
\end{tabular}




\section{S3 Reactor Specifications}

In this section we present the reactor sizing data, operating condition, and catalyst properties used in the case studies.

Methanol Synthesis Reactor: Reactor design parameters of a industrial scale Lurgi-type reactor are presented in Table S6 which is adopted from Arora et al. ${ }^{7}$

Table S6: Design parameters of methanol synthesis reactor.

\begin{tabular}{lc}
\hline Parameters & Value \\
\hline Number of tubes & 2962 \\
Tube length $(\mathrm{m})$ & 7.022 \\
Tube diameter $(\mathrm{cm})$ & 3.675 \\
Catalyst & $\mathrm{Cu}-\mathrm{ZnO}-\mathrm{Al}_{2} \mathrm{O}_{3}$ \\
Catalyst packing density $\left(\mathrm{kg}_{\text {cat }} \cdot \mathrm{m}^{-3}\right)$ & 1100 \\
Catalyst bed voidage $(\%)$ & 0.38 \\
\hline
\end{tabular}

Partial Oxidation Reactor: Specification of the partial oxidation reactor is presented in Table S7.

Table S7: Design parameters of POM reactor.

\begin{tabular}{lcc}
\hline Parameters & Value & Reference \\
\hline Number of tubes & 2000 & \\
Reactor length $(\mathrm{m})$ & 6 & Tsai et al. ${ }^{8}$ \\
Tube diameter $(\mathrm{cm})$ & 2.5 & Tsai et al. ${ }^{8}$ \\
Catalyst packing density $\left(\mathrm{kg}_{\mathrm{c} a t} . \mathrm{m}^{-3}\right)$ & 2100 & $\mathrm{Ji}^{\mathrm{et} \mathrm{al} .}$ \\
Catalyst bed voidage $(\%)$ & 0.43 & Ji et al. $^{9}$ \\
Reactor inlet temperature $\left({ }^{\circ} \mathrm{C}\right)$ & 500 & De Smet et al. ${ }^{5}$ \\
Reactor pressure (bar) & 25 & Alsuhaibani et al. ${ }^{10}$ \\
\hline
\end{tabular}

As in both case studies the reactor configurations are fixed, the total catalyst amount is determined from the reactor length, tube number, tube diameter, catalyst packing density, catalyst porosity, etc. by Eq. S138. Similarly, the total surface area of membrane tubes are also determined from the reactor geometry by Eqs. S140-S141.

\section{S4 Surrogate Models of Thermodynamic Properties}

We have used quadratic surrogate models to predict thermodynamic properties such as specific enthalpies and saturation pressures. The model parameters are obtained by fitting thermodynamic data obtained from rigorous simulation or experiment. One such example of quadratic models is shown below: 


$$
\begin{gathered}
H_{k}^{\text {vap }}=H_{k}^{v a p, a} T_{i, j}^{2}+H_{k}^{v a p, b} T_{i, j}+H_{k}^{v a p, c}, \quad i \in \mathcal{I}, j \in \mathcal{J}, k \in \mathcal{K}, \\
H_{k}^{l i q}=H_{k}^{l i q, a} T_{i, j}^{2}+H_{k}^{l i q, b} T_{i, j}+H_{k}^{l i q, c}, \quad i \in \mathcal{I}, j \in \mathcal{J}, k \in \mathcal{K}, \\
P_{k}^{s a t}=P_{k}^{a} T_{i, j}^{2}+P_{k}^{b} T_{i, j}+P_{k}^{c}, \quad i \in \mathcal{I}, j \in \mathcal{J}, k \in \mathcal{K} .
\end{gathered}
$$

For example, as methanol synthesis and POM are both gas phase reactions, block phases are fixed as gas/vapor phase in both case studies. Therefore, saturation pressure is not estimated to determine the block phases and only the vapor phase specific enthalpy is considered in energy balance calculation. Specific enthalpy of each species are estimated from ASPEN PLUS ${ }^{\circledR}$ between $300 \mathrm{~K}$ and $1600 \mathrm{~K}$ from a reference temperature of $298 \mathrm{~K}$. Then it is fitted to a polynomial function to estimate the parameters of the surrogate model for $H_{k}^{v a p}$, which are presented in Table S8.

Table S8: Vapor phase specific enthalpy parameters.

\begin{tabular}{cccc} 
& $H_{k}^{v a p, a}\left[\frac{k J}{k m o l . K^{2}}\right]$ & $H_{k}^{v a p, b}\left[\frac{k J}{k m o l . K}\right]$ & $H_{k}^{v a p, c}\left[\frac{k J}{k m o l}\right]$ \\
\hline $\mathrm{CH}_{4}$ & 0.0224 & 26.481 & -9902.0 \\
$\mathrm{O}_{2}$ & 0.0029 & 28.778 & -8839.3 \\
$\mathrm{~N}_{2}$ & 0.0028 & 26.946 & -8271.3 \\
$\mathrm{H}_{2}$ & 0 & 29.210 & -8722.5 \\
$\mathrm{CO}$ & 0.0029 & 27.194 & -8388.4 \\
$\mathrm{CO}$ & 0.0075 & 38.356 & -12112 \\
$\mathrm{H}_{2} \mathrm{O}$ & 0.0061 & 29.125 & -9209.5 \\
$\mathrm{CH}_{3} \mathrm{OH}$ & 0.0401 & 19.675 & -9423.9
\end{tabular}

As the temperature bounds are known, these simple surrogate models offer higher accuracy. Figure $\mathrm{S} 1$, shows the $R^{2}$ values of the fitted models. 

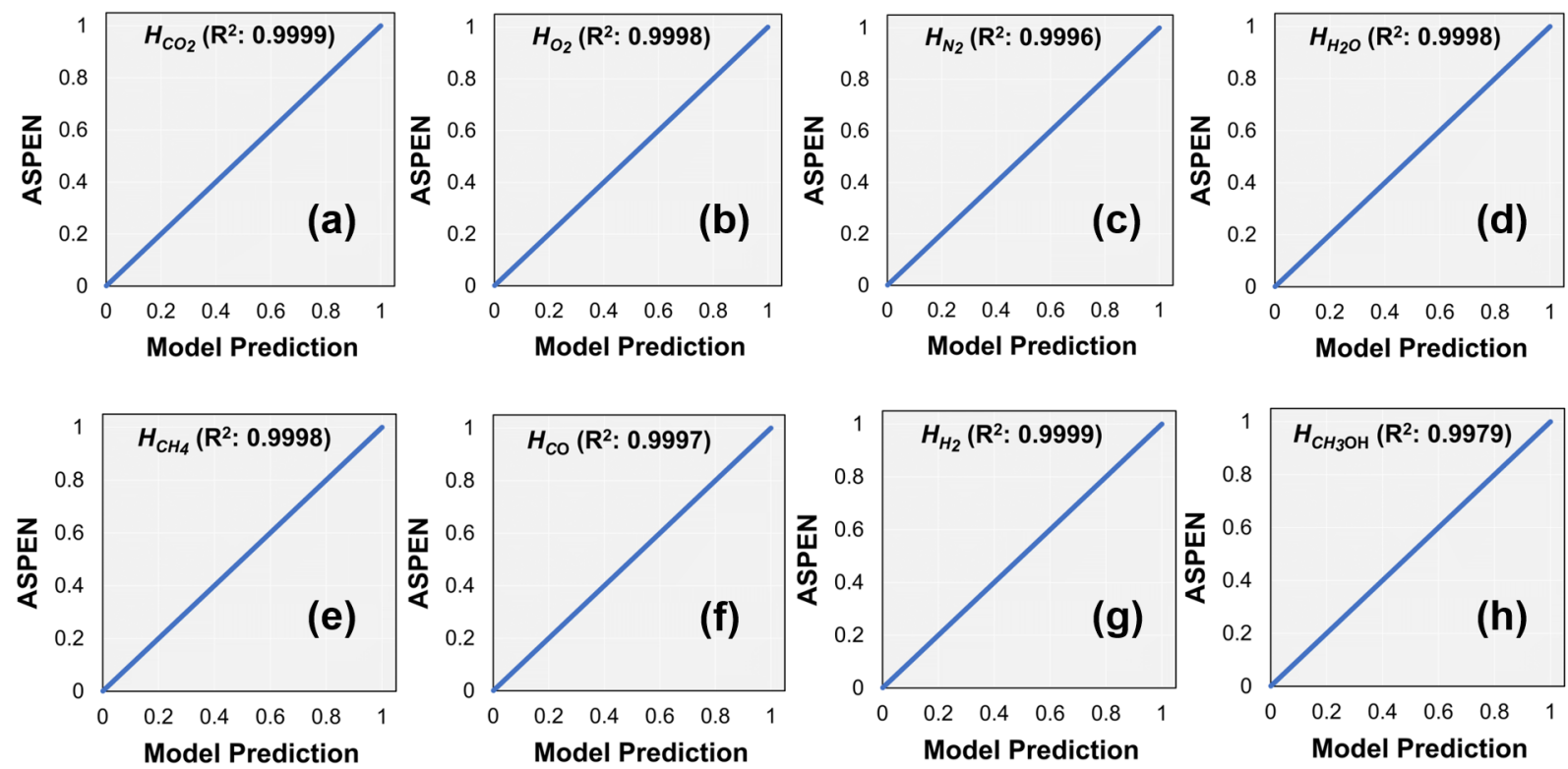

Figure S1: Accuracy of surrogate models for enthalpy estimation for (a) $\mathrm{CO}_{2}$, (b) $\mathrm{O}_{2}$, (c) $\mathrm{N}_{2}$, (d) $\mathrm{H}_{2} \mathrm{O}$, (e) $\mathrm{CH}_{4}$, (f) $\mathrm{CO}$, (g) $\mathrm{H}_{2}$, and (h) $\mathrm{CH}_{3} \mathrm{OH}$ between temperature range of $300 \mathrm{~K}$ to $1600 \mathrm{~K}$.

\section{S5 in silico Validation of Thermodynamic Property Models}

In the main manuscript, we discuss about the simulation accuracy of SPICE_MARS and compare the simulation results of an isothermal and an adiabatic methanol synthesis reactor with ASPEN PLUS ${ }^{\circledR}$ simulation. Here, we present some additional comparison to show the accuracy of the surrogate models used to estimate thermodynamic properties. Similar to the SPICE_MARS flow sheet (Figure S2a), the first unit in ASPEN PLUS ${ }^{\circledR}$ flow sheet (Figure S2b) is a compressor that compresses the syngas to the reactor operating pressure. After the compressor, the syngas is then heated in a heater before it enters to the synthesis reactor.

(a)

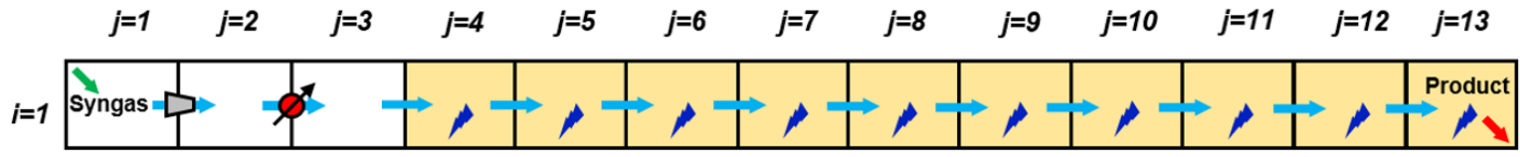

(b)

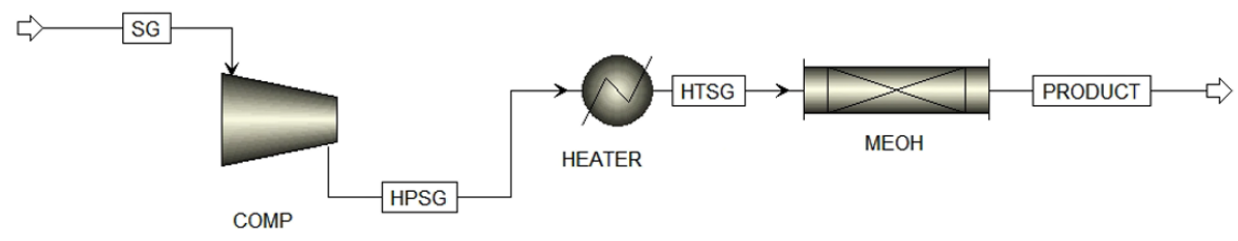

Figure S2: Methanol synthesis process simulation, (a) $1 \times 13$ building block superstructure considered in SPICE_MARS, and (b) corresponding ASPEN PLUS ${ }^{\circledR}$ flow sheet. 
From Table S9 we can see that, for the isothermal reactor simulation, the compression work reported by SPICE_MARS is $4704 \mathrm{~kW}$, wheres, ASPEN PLUS ${ }^{\circledR}$ requirement is $4676 \mathrm{~kW}$ and 4754 kW for Ideal Gas and Peng-Robinson model, respectively. The outlet temperatures of compressor in SPICE_MARS and in both models of ASPEN PLUS ${ }^{\circledR}$ are 397 K. Heating utility $(\mathrm{kW})$ requirement in SPICE_MARS is $0.31 \%$ lower than that of the Ideal Gas model and $2.26 \%$ lower than that of the Peng-Robinson model. Similarly, the cooling utility $(\mathrm{kW})$ requirement in SPICE_MARS is $3.94 \%$ and $6.97 \%$ lower than Ideal Gas and Peng-Robinson model, respectively.

Table S9: Comparison between SPICE_MARS and ASPEN PLUS ${ }^{\circledR}$ simulation of isothermal and adiabatic methanol synthesis reactor.

\begin{tabular}{|c|c|c|c|c|c|}
\hline Variables & SPICE_MARS & $\begin{array}{l}\text { ASPEN PLUS }{ }^{\circledR} \\
\quad \text { (Ideal Gas) }\end{array}$ & Deviation (\%) & $\begin{array}{l}\text { ASPEN PLUS }{ }^{\circledR} \\
\quad(\text { Peng-Rob) }\end{array}$ & Deviation (\%) \\
\hline \multicolumn{6}{|c|}{ Isothermal reactor } \\
\hline Compressor work $(\mathrm{kW})$ & 4704 & 4676 & -0.59 & 4754 & 1.06 \\
\hline Compressor outlet temperature $(\mathrm{K})$ & 397 & 397 & 0 & 397 & 0 \\
\hline Heating duty in heater $(\mathrm{kW})$ & 6443 & 6463 & 0.31 & 6589 & 2.26 \\
\hline Cooling duty in reactor $(\mathrm{kW})$ & 5935 & 6169 & 3.94 & 6349 & 6.97 \\
\hline \multicolumn{6}{|c|}{ Adiabatic reactor } \\
\hline Compressor work $(\mathrm{kW})$ & 2872 & 2862 & -0.35 & 2899 & 0.94 \\
\hline Compressor outlet temperature $(\mathrm{K})$ & 366 & 366 & 0 & 366 & 0 \\
\hline Heating duty in heater $(\mathrm{kW})$ & 8275 & 8278 & 0.03 & 8435 & 1.93 \\
\hline Maximum reactor temperature $(\mathrm{K})$ & 551 & 551 & 0 & 551 & 0 \\
\hline
\end{tabular}

On the other hand, for the adiabatic reactor simulation, SPICE_MARS's prediction of compressor work is $2872 \mathrm{~kW}$ which has a $-0.35 \%$ deviation from Ideal Gas and $0.94 \%$ deviation from Peng-Robinsion model. The compressor outlet temperature in SPICE_MARS simulation is $366 \mathrm{~K}$ which matches with the simulation results of ASPEN PLUS ${ }^{\circledR}$ models. The heating duty (kW) requirements found in SPICE_MARS is also comparable with both ASPEN PLUS ${ }^{\circledR}$ simulations. Moreover, the maximum reactor temperature reported by the SPICE_MARS matches with the ASPEN PLUS ${ }^{\circledR}$ simulations.

\section{S6 Capital Cost Functions for Total Annual Cost (TAC)}

In this section, we provide the detailed version of the capital cost functions used for estimating the TAC in the second case study. The capital cost of the POM reactor ${ }^{10}$ is calculated from the flow rate $(\mathrm{mol} / \mathrm{s})$ of the products by

$$
\text { Reactor }(\$)=31.02 \times 10^{6}\left[\frac{\sum_{i, j \in \mathcal{R} \mathcal{X} \mathcal{N} \mathcal{B}} \sum_{k \in \mathcal{K}} \sum_{p \in \mathcal{P} \mathcal{S}} N_{i, j, k, p}}{5333}\right]^{0.67} \times \frac{I_{2019}}{I_{2009}} .
$$

Cost of membrane tubes ${ }^{11}$ are calculated based on membrane surface area $\left(\mathrm{m}^{2}\right)$ by 


$$
\text { Membrane }(\$)=130000\left[\frac{\sum_{i \in \mathcal{I}} \sum_{j \in \mathcal{J}} \sum_{(s, m) \in \mathcal{S} \mathcal{M}}\left(A_{i, j, s, m}^{F}+A_{i, j, s, m}^{R}\right)}{560}\right] \times \frac{I_{2019}}{1000} \text {. }
$$

Here, the term $I_{\text {year }}$ represents the Chemical Engineering Plant Cost Index (CEPCI). Compressor capital cost $^{12}$ is calculated based on the power requirement $(\mathrm{kW})$,

$$
\text { Compressor }(\$)=5840\left[\sum_{i \in \mathcal{I}} \sum_{j \in \mathcal{J}} W_{i, j}^{\text {comp }}\right]^{0.82} \text {. }
$$

The full form of the TAC is as follows,

$$
\begin{aligned}
\mathrm{TAC}= & t^{\text {opt }} \times \sum_{i \in \mathcal{I}} \sum_{j \in \mathcal{J}} \sum_{k \in \mathcal{K}}\left(U C^{\text {Oxygen }} M_{i, j, k, \text { Oxygen }}+U C^{\text {Steam }} M_{i, j, k, \text { Steam }}\right) \\
& +t^{\text {opt }} \times \frac{U C^{\text {elec }}}{\eta_{c}} \sum_{i \in \mathcal{I}} \sum_{j \in \mathcal{J}} W_{i, j}^{\text {comp }}+0.154 \times 5840\left[\sum_{i \in \mathcal{I}} \sum_{j \in \mathcal{J}} W_{i, j}^{\text {comp }}\right]^{0.82} \\
& +0.154 \times 31.02 \times 10^{6}\left[\frac{\sum_{i, j \in \mathcal{R} \mathcal{X} \mathcal{N} \mathcal{B}} \sum_{k \in \mathcal{K}} \sum_{p \in \mathcal{P} \mathcal{S}} N_{i, j, k, p}}{5333}\right]^{0.67} \times \frac{599.3}{521.9} \\
& +130000\left[\frac{\sum_{i \in \mathcal{I}} \sum_{j \in \mathcal{J}} \sum_{(s, m) \in \mathcal{S} \mathcal{M}}\left(A_{i, j, s, m}^{F}+A_{i, j, s, m}^{R}\right)}{560}\right] \times \frac{599.3}{1000} .
\end{aligned}
$$

Here, $t^{\text {opt }}$ represents the yearly operating time in second considering 365 days of operation. Raw material costs of only pure $\mathrm{O}_{2}$ and steam is considered in the TAC as methane is the common feed to both $\mathrm{CR}$ and MR with a fixed flow rate. On the other hand, air is available at atmospheric conditions. The cost of pure $\mathrm{O}_{2}$, steam, and electricity is $\$ 100 /$ ton, $\$ 5 /$ ton, and $\$ 0.05 / \mathrm{kWh}$, respectively and taken from Alsuhaibani et al. ${ }^{10}$ The efficiency of the air compressor is considered to be $80 \%$. Capital recovery factor $\left(\alpha^{C R F}\right)$ for the air compressor and POM reactor is considered to be $15.4 \%$. However, the membrane tubes are considered to be replaced every year, thus $\alpha^{C R F}$ is not used to estimated the annualized cost of the membrane tubes.

\section{S7 Selectivity and Syngas Ratio}

The selectivity of $\mathrm{CO}, \mathrm{CO}_{2}$, and $\mathrm{H}_{2}$ and the syngas ratio (SR) of the POM reactor is calculated by the following equations:

CO selectivity:

$$
\mathrm{S}_{\mathrm{CO}}=\frac{\mathrm{F}_{\mathrm{CO}}^{\mathrm{out}}}{\mathrm{F}_{\mathrm{CH}_{4}}^{\text {in }}-\mathrm{F}_{\mathrm{CH}_{4}}^{\text {out }}} \times 100
$$


$\mathrm{CO}_{2}$ selectivity:

$$
\mathrm{S}_{\mathrm{CO}_{2}}=\frac{\mathrm{F}_{\mathrm{CO}_{2}}^{\text {out }}}{\mathrm{F}_{\mathrm{CH}_{4}}^{\mathrm{in}}-\mathrm{F}_{\mathrm{CH}_{4}}^{\text {out }}} \times 100
$$

$\mathrm{H}_{2}$ selectivity:

$$
\mathrm{S}_{\mathrm{H}_{2}}=\frac{\mathrm{F}_{\mathrm{H}_{2}}^{\text {out }}}{2 \times\left(\mathrm{F}_{\mathrm{CH}_{4}}^{\mathrm{in}}-\mathrm{F}_{\mathrm{CH}_{4}}^{\text {out }}\right)} \times 100
$$

Syngas ratio:

$$
\mathrm{SR}=\frac{\mathrm{F}_{\mathrm{H}_{2}}^{\text {out }}}{\mathrm{F}_{\mathrm{CO}}^{\text {out }}}
$$

Here, $\mathrm{F}_{\mathrm{CH}_{4}}^{\mathrm{in}}$ and $\mathrm{F}_{\mathrm{CH}_{4}}^{\text {out }}$ are the inlet and the outlet flow rate of $\mathrm{CH}_{4}$ to and from the reactor. Similarly, $\mathrm{F}_{\mathrm{CO}}^{\text {out }}, \mathrm{F}_{\mathrm{CO}}^{\text {out }}, \mathrm{F}_{\mathrm{H}_{2}}^{\text {out }}$ are the outlet flow rate of $\mathrm{CO}, \mathrm{CO}_{2}$, and $\mathrm{H}_{2}$, respectively.

\section{References}

[1] Demirel, S. E.; Li, J.; Hasan, M. M. F. Systematic process intensification using building blocks. Computers \& Chemical Engineering 2017, 105, 2-38.

[2] Bussche, K. V.; Froment, G. A steady-state kinetic model for methanol synthesis and the water gas shift reaction on a commercial $\mathrm{Cu} / \mathrm{ZnO} / \mathrm{Al} 2 \mathrm{O} 3$ Catalyst. Journal of Catalysis 1996, 161, $1-10$.

[3] De Groote, A. M.; Froment, G. F. Simulation of the catalytic partial oxidation of methane to synthesis gas. Applied Catalysis A: General 1996, 138, 245-264.

[4] Trimm, D. L.; Lam, C.-W. The combustion of methane on platinum-alumina fibre catalysts-I: Kinetics and mechanism. Chemical Engineering Science 1980, 35, 1405-1413.

[5] De Smet, C.; De Croon, M.; Berger, R.; Marin, G.; Schouten, J. Design of adiabatic fixed-bed reactors for the partial oxidation of methane to synthesis gas. Application to production of methanol and hydrogen-for-fuel-cells. Chemical Engineering Science 2001, 56, 4849-4861.

[6] Xu, J.; Froment, G. F. Methane steam reforming, methanation and water-gas shift: I. Intrinsic kinetics. AIChE Journal 1989, 35, 88-96.

[7] Arora, A.; Iyer, S. S.; Bajaj, I.; Hasan, M. M. F. Optimal methanol production via sorptionenhanced reaction process. Industrial \& Engineering Chemistry Research 2018, 57, 1414314161.

[8] Tsai, C.-Y.; Ma, Y. H.; Moser, W. R.; Dixon, A. G. Modeling and simulation of a nonisothermal catalytic membrane reactor. Chemical Engineering Communications 1995, 134, 107-132. 
[9] Ji, P.; Van der Kooi, H.; de Swaan Arons, J. Simulation and thermodynamic analysis of conventional and oxygen permeable CPO reactors. Chemical Engineering Science 2003, 58, 2921-2930.

[10] Alsuhaibani, A. S.; Afzal, S.; Challiwala, M.; Elbashir, N. O.; El-Halwagi, M. M. The impact of the development of catalyst and reaction system of the methanol synthesis stage on the overall profitability of the entire plant: A techno-economic study. Catalysis Today 2020, 343, 191-198.

[11] Woods, D. R. Rules of thumb in engineering practice; John Wiley \& Sons, 2007.

[12] Douglas, J. M. Conceptual design of chemical processes; McGraw-Hill New York, 1988; Vol. 1110. 